\title{
Membrane insertion of Cavin1 facilitates caveolae assembly
}

Kang-cheng Liu ${ }^{1}$, Hudson Pace ${ }^{1}$, Elin Larsson ${ }^{1}$, Shakhawath Hossain ${ }^{2}$, Aleksei Kabedev $^{2}$, Jagan Mohan ${ }^{1, \dagger}$, Christel A. S. Bergström², Christian Schwieger ${ }^{3,}$, Madlen Hubert $^{2}$ and Richard Lundmark ${ }^{1, *}$

${ }^{1}$ Integrative Medical Biology, Umeå University, 901 87, Umeå, Sweden

${ }^{2}$ Department of Pharmacy, Uppsala University, Uppsala Biomedical Center, P.O Box 580, SE-751 23 Uppsala, Sweden

${ }^{3}$ Institute of Chemistry, Martin Luther University Halle-Wittenberg, 06120 Halle (Saale), Germany

${ }^{\dagger}$ Current address: Membrane Biochemistry and Transport, Institute Pasteur, 28 rue du Dr Roux, 75015 Paris, France.

$\$$ Current address: Interdisciplinary Research Center HALOmem, Institute of Biochemistry and Biotechnology, Martin Luther University Halle-Wittenberg, 06120 Halle (Saale), Germany

*Corresponding author: Richard Lundmark, Department of Integrative Medical Biology, Umeå University, 90187 Umeå, Sweden, phone: +46 706202464, email: richard.lundmark@umu.se 


\begin{abstract}
Caveolae are small cell surface invaginations, important for control of membrane tension, signaling cascades and lipid sorting. Their formation is coupled to the lipiddependent oligomerization of the proteins Caveolin1 and Cavin1, which are essential for membrane curvature generation. Yet, the mechanistic understanding of how Cavin1 assembles at the membrane interface is lacking. Here, we used model membranes combined with biophysical techniques to show that Cavin1 inserts into membranes. We found that the helical region 1 (HR1) initiated membrane binding via electrostatic interactions, which is further enforced by partial helical insertion in a $\mathrm{PI}(4,5) \mathrm{P}_{2}-$ dependent process mediated by the disordered region 1 (DR1). In agreement with this, the DR1 was found important for the co-assembly of Cavin1 with Caveolin1 in living cells. We propose that DR1 and HR1 of Cavin1 constitute a novel membrane interacting unit facilitating dynamic rounds of assembly and disassembly of Cavin1 at the membrane.
\end{abstract}

\title{
TEASER
}

Assembly of the caveolae coat proteins involve lipid-driven partial insertion of Cavin1 into the membrane bilayer

\section{INTRODUCTION}

Caveolae are small bulb shaped invaginations $(50-80 \mathrm{~nm})$ of the plasma membrane. They are highly abundant in adipocytes, fibroblasts, muscle and endothelial cells and are important in various physiological processes like regulation of membrane tension, lipid metabolism and cellular signaling $(1,2)$. Lack or dysfunction of caveolae are connected with severe human diseases such as muscular dystrophy, cardiomyopathy and lipodystrophy. Caveolae formation requires the presence of special lipids compositions and the coat components Caveolin1 (CAV1) and Cavin1(3). These proteins are universal structural elements and knock out of either of these proteins leads to loss of caveolae $(1,2)$. CAV1 belongs to a family of integral membrane proteins (CAV1-3) where both the $\mathrm{N}$ - and C-terminal protrude into the cytoplasm. Cavin1 belongs to a family comprised of four different proteins (Cavin1-4) which exhibit tissue 
specific expression patterns (3). Caveolae are considered to be specific lipid domains of the plasma membrane, enriched in cholesterol and sphingolipids $(1,2)$. These lipids not only accumulate in caveolae, but are actively sequestered (4). Lipid mapping in cells showed that both CAV1 and Cavin1 recruit specific lipid species to caveolae hereby acting synergistically to generate the unique lipid nano-environment of caveolae (5). Electron microscopy studies on caveolae have revealed a striated protein coat lining, which is believed to comprise CAV1 and the Cavin proteins $(6,7)$. CAV1 has been shown to form high order $8 \mathrm{~S}$ oligomers in membranes following cholesterol binding (8) and single particle cryo-EM analysis has revealed a toroidal structure assembled by wedge-like sections of CAV1 monomers (9). The Cavin proteins are thought to assemble with the CAV1 8S complexes to form 60S and 80S complexes building up the caveolae coat (10).

The Cavin proteins share a common pattern in their domain structure containing negatively charged disordered regions (DRs) interspersed with more positively charged helical regions (HRs) (domain structure of Cavin1 illustrated in Figure 1A). The crystal structures of mouse and zebrafish HR1 (4QKV and 4QKW, respectively) revealed an extended $\alpha$-helical trimeric coiled-coil structure (11). The HR1 domain of Cavin1 has been shown to mediate trimeric homo-oligomerization and formation of heterocomplexes with either Cavin2 or Cavin3 in solution $(11,12)$. Analysis of the caveolae coat stoichiometry predicted that Cavin1 forms a complex with either Cavin2 or Cavin 3 in a ratio of $2: 1$ or $3: 1 \quad(6,7,11,12)$. Yet, the precise stoichiometry remains to be elucidated. Cryo-electron microscopy studies of the 60S and 80S CAV1 and Cavins complexes proposed an architecture composed of an inner coat of polygonal units of Caveolins, and an outer Cavins coat aligned along the edges of the inner polyhedral cage $(13,14)$. The models propose that Cavin arranges into a web-like architecture composed by an interbranched trimeric complex (13) or alternatively that the Cavins are stacked in rod-like trimers (14).

The assembly of higher ordered complexes of CAV1 and Cavin1 is highly influenced by lipids and recent data suggest that the caveolae coat composition is flexible and adapts the local lipid environment (15). Besides cholesterol and sphingolipids caveolae are also enriched in negatively charged lipids such as phosphatidylserine (PS) and 
phosphatidylinositol $(4,5)$ bisphosphate $\left(\mathrm{PI}(4,5) \mathrm{P}_{2}\right)(16)$. In vitro studies have shown that Cavin1 binds both PS and PI(4,5) $\mathrm{P}_{2}$ supposedly via electrostatic interactions (11, 17). The positively charged amino acids (Lys115, Arg117, Lys118, Lys124, Arg127) in the HR1 domain mediate specific binding to $\mathrm{PI}(4,5) \mathrm{P}_{2}(11)$, whereas a repeated sequence of 11 amino acids of the HR2 domain, identified as undecad repeat (UC1), was shown to bind PS and regulate caveolar stability (17). Interestingly, the assembly of both CAV1 and Cavin1 was found to be dependent on the acyl chain composition of PS, suggesting that the interactions of these proteins with the hydrophobic region of the membrane is important (5).

The shape of the HR1 domain is very different from proteins inducing curvature via scaffolding and no protruding hydrophobic residues that would enable membrane insertion exist. It has been proposed that Cavin1 might drive membrane curvature by molecular crowding via weak electrostatic interactions between the DR and HR regions (18). Removal of the DR1 domain was shown to cause an irreversible gel formation in vitro and to influence caveolae formation as well as trafficking through the endosomal system in cells (18). However, it is still not understood how the unique striped or spiral pattern of the caveolae coat is assembled and what intermolecular forces join the molecular components together.

In this work, we address the detailed mechanism by which Cavin1 binds and assembles at the lipid interface using model membranes in combination with a variety of biophysical techniques. We found that Cavin1 inserted into the membrane via the HR1 domain, assisted by the DR1 domain, in a $\mathrm{PI}(4,5) \mathrm{P}_{2}$-mediated process. Furthermore, we report that interference with this insertion mechanism affect the co-assembly of Cavin1 and CAV1 in cells during caveolae formation. 


\section{RESULTS}

\section{Cavin1 inserts into the membrane providing stable membrane association.}

To characterize the mechanism of membrane driven assembly of Cavin1, we purified full-length Cavin1 from mammalian HEK cells (fig. S1, A and B). Post-translational modifications of the purified protein were characterized by tandem mass spectrometry. The data showed that Cavin 1 was ubiquitinated within the DR3 domain at residue serine 302 (Fig. 1A and fig. S1C), which is distinct from the previously proposed lysineubiquitination involved in Cavin1 degradation (19). Furthermore, our data showed that Cavin1 was phosphorylated at 15 different sites primarily located in clusters in the HR2 domain and the disordered regions (Fig. 1A and fig. S1C). These modifications likely explain the migratory behavior of Cavin1 on SDS-PAGE gel electrophoresis, where it runs at $60 \mathrm{kDa}$ instead of the expected $43 \mathrm{kDa}$ (fig. S1B). To verify that the purified protein could bind membranes, we used liposomes composed of DOPC:DOPE:PI(4,5)P2 (55:45:5 mol\%). Analysis of liposome binding using a cosedimentation assay showed that Cavin1 bound to such vesicles with relatively high positive membrane curvature (Fig. 1B). To address the membrane association in a system independent of membrane curvature, and where binding over time could be quantified, we generated flat supported lipid bilayers (SLBs) composed of POPC:PI(4,5) $\mathrm{P}_{2}(95: 5 \mathrm{~mol} \%)$ on a glass surface. Quartz crystal microbalance with dissipation monitoring (QCM-D) has been used extensively to monitor SLB formation (20-22) as it provides a distinct signature of changes in the adsorbed mass (frequency, $\Delta \mathrm{F}$ ) and stiffness (dissipation, $\Delta \mathrm{D}$ ) of the surface (Fig. 1C). In short, as the relatively soft liposomes adsorb to the rigid substrate, the frequency drops and dissipation increases until the surface crowding of liposomes reaches a critical point and liposomal rupture becomes the predominant process (Fig. 1C, scheme). The transition from spherical liposome to planar SLB increases the stiffness of the adsorbed lipid assembly (drop in dissipation D) and involves the release of luminal content (increase in frequency F). Once the SLB is fully formed and a steady-state is reached, the dissipation and frequency values provide a signature for the SLB that can be used to assess its quality. Addition of purified Cavin 1 to the SLB resulted in a steep decrease in frequency and increase in dissipation representing adsorption of the protein to the SLB (Fig. 1D). When the system was rinsed with buffer $(150 \mathrm{mM} \mathrm{NaCl})$, we noticed a slow but steady release of Cavin1 from the SLB surface, indicating that the binding is at least partially 
reversible. We next tested if Cavin1 was adsorbed to the SLB through electrostatic interactions by treating the system with increased salt concentration. The change to 300 $\mathrm{mM} \mathrm{NaCl}$ caused a sudden shift in both the frequency and dissipation, but was reversed upon switching back to the initial buffer. We found that higher concentration of salt did not cause additional protein desorption from the SLB beyond the slow rate observed prior to the high salt treatment (Fig. 1D, grey dotted line). This resistance to high salt treatment suggests that, once bound to the membrane, Cavin1 was not solely interacting with the SLB through electrostatic interactions, but rather in combination with hydrophobic forces.

To decipher if Cavin1 would insert into membranes, we probed the adsorption to a monolayer suspended at an air/water interface where changes in the lateral pressure of the monolayer can be measured. The surface pressure $(\pi)$ is directly related to the lateral cohesion of molecules and initial surface pressure values $\left(\pi_{0}\right)$ are related to the lateral packing density of the lipids before protein interactions. We prepared lipid films of DOPC:DOPE:PI(4,5)P 2 (55:45:5 mol\%) on a buffer surface and injected Cavin1 underneath the lipid monolayer into the subphase (Fig. 1E). The rapid increase in surface pressure seen following protein injection indicated that Cavin1 instantly adsorbed to and inserted into the lipid monolayer (Fig. 1E) (23). To get further insights into binding mechanisms, we measured Cavin1 adsorption at various initial surface pressures $\left(\pi_{0}\right)$ and monitored surface pressure variation $(\Delta \pi)$ induced by protein/lipid interaction (fig. S1D). Linear regressions of $\Delta \pi=\mathrm{f}\left(\pi_{0}\right)$ provides a synergy factor $(a)$ that corresponds to the slope $>1$ and describes the protein affinity for the lipid monolayer (Fig. 1F) The positive synergy factor value of $a=0.41$ for Cavin1 indicated the existence of strong protein/lipid interactions $(24,25)$. This is further supported by a high maximum insertion pressure (MIP) value of $36 \pm 1.9 \mathrm{mN} \mathrm{m}^{-1}$ obtained by extrapolation of the adsorption data to the $\mathrm{x}$-axis. Proteins with MIP values above the estimated monolayer/bilayer equivalence pressure $\left(\sim 30 \mathrm{mN} \mathrm{m}^{-1}\right)$ are considered to be well incorporated into the lipid layer (26). Our data indicated a high extent of Cavin1 membrane insertion, likely due to a combination of both electrostatic and hydrophobic forces.

\section{The N-terminal region of Cavin1 inserts into membranes in a $\mathrm{PI}(4,5) \mathrm{P}_{2}$ dependent manner.}


To identify the region required for membrane insertion of Cavin1, we expressed and purified the N-terminal part (1-190) and the C-terminal part (190-392) of Cavin1 as recombinant proteins from bacteria. By performing a co-sedimentation assay we found that the truncated Cavin1 (1-190) variant bound membranes equally well as the fulllength protein (Fig. 2A and Fig. 1B, respectively). However, as previously shown, the C-terminal region (191-392) was unable to bind to liposomes composed of DOPC:DOPE:PI(4,5)P 2 (17). To test whether the 1-190 region of Cavin1 was responsible for the observed membrane insertion, we performed adsorption experiments using lipid monolayers. The data revealed a remarkable increase in surface pressure following injection of Cavin1 (1-190) underneath a DOPC:DOPE:PI(4,5) $\mathrm{P}_{2}$ film, showing that this part of the protein does insert (Fig. 2B). While the synergy factor of $a=0.43$ was similar to that of Cavin1, the MIP was determined to be $49 \pm 2.2 \mathrm{mN}$ $\mathrm{m}^{-1}$ in comparison to $36 \pm 1.9 \mathrm{mN} \mathrm{m}^{-1}$, confirming that Cavin1 (1-190) has a high affinity for the lipid interface and is strongly incorporated into the monolayer (Fig. 2C and fig. S2B).

To further verify the presence of the protein at the interface and investigate its effect on the membrane organization, we used infrared reflection-absorption spectroscopy (IRRAS). IRRA spectra of the pure lipid films consisting of either DOPC:DOPE:PI(4,5)P2 (Fig. 2D) or DOPC:DOPE (fig. S2C) displayed characteristic $\mathrm{C}=\mathrm{O}$ vibrational bands at $\sim 1730 \mathrm{~cm}^{-1}$ originating from the ester group of the lipids. After injection of Cavin1 (1-190), the presence of the protein at the air/buffer interface was indicated by the amide I' band with a maximum at $\sim 1640 \mathrm{~cm}^{-1}$ (Fig. $2 \mathrm{D}$ and fig. $\mathrm{S} 2 \mathrm{C}$ ). The frequency of the carbonyl vibration is sensitive to H-bond formation and thus provides information on the lipid hydration at the hydrophilic/hydrophobic interface (27). Because the IRRA spectra indicated a shift of the $\mathrm{C}=\mathrm{O}$ vibrational bands towards higher wavenumbers after protein injection, we subjected a larger set of collected spectra to principal component analysis (PCA) to determine if the presence of the protein at the interface affected lipid hydration. PCA revealed that the major differences between the analyzed spectra (fig. S2D) are indeed due to different extents in hydration (Fig. 2E). The first principal component (PC 1), clearly indicated a decrease of the spectral component at $1645 \mathrm{~cm}^{-1}$ (less hydrated $\mathrm{C}=\mathrm{O}$ groups) and a concomitant increase of the spectral component at $1715 \mathrm{~cm}^{-1}$ (more hydrated $\mathrm{C}=\mathrm{O}$ groups) (fig. S2E). The analyzed set of spectra clearly separated into two groups: more 
hydrated carbonyls in pure lipid monolayers and less hydrated carbonyl groups in presence of Cavin1 (1-190) (Fig. 1E). Interestingly, the presence of $\mathrm{PI}(4,5) \mathrm{P}_{2}$ in the lipid mixture did not affect the monolayer hydration, neither in absence nor in presence of Cavin1 (1-190). Moreover, IRRA spectra of pure lipid films at the surface pressure of injection $\left(22 \mathrm{mN} \mathrm{m}^{-1}\right)$ and after protein adsorption $\left(36 \mathrm{mN} \mathrm{m}^{-1}\right)$ (fig. $\mathrm{S} 2 \mathrm{~F}$ ) appeared to be similar. This indicated that lipid dehydration is not merely an effect of increasing surface pressure following Cavin1 injection but can clearly be assigned to insertion of Cavin1 (1-190) into the lipid head group region and the concomitant replacement of hydration water.

Next, we determined whether $\mathrm{PI}(4,5) \mathrm{P}_{2}$ influenced the quantity of protein adsorbed to the lipid monolayer. While the position of the $\mathrm{C}=\mathrm{O}$ and amide I' bands were comparable, the intensity of amide I', which correlates to the amount of adsorbed protein, was increased when $\mathrm{PI}(4,5) \mathrm{P}_{2}$ was present in the lipid layer (Fig. 2D and fig. $\mathrm{S} 2 \mathrm{C})$. To assess the amount of inserted protein per lipid molecule, the ratios of integral intensities of amide I'/C=O of various IRRA spectra recorded at different angles of incidence were calculated for both lipid monolayers after Cavin1 (1-190) adsorption (Fig. 2F). The data showed significantly higher protein/lipid ratios for DOPC:DOPE:PI $(4,5) \mathrm{P}_{2}$ films (amide I'/ $\left./ \mathrm{C}=\mathrm{O}\right)=5.71$ vs. $3.24,{ }^{*} p<0.05$ ) indicating a strong correlation between the presence of $\mathrm{PI}(4,5) \mathrm{P}_{2}$ and the amount of adsorbed Cavin1 (1-190) (28).

\section{Membrane insertion is facilitated by the combined DR1 and HR1 domain.}

To further examine the mechanism for how the N-terminal region (1-190) interacts and inserts into the membrane we wanted to know how removal of the DR1 and or DR2 regions would affect this process. The Cavin1 N-terminal region (1-190), DR1 and HR1 (1-155), HR1 (44-155) and HR1 and DR2 (44-190) were expressed and purified from E. coli (fig. S3A). Using far-UV circular dichroism (CD) spectroscopy, we found that the purified constructs all exhibited a predominantly $\alpha$-helical CD profile with typical minima at 208 and $222 \mathrm{~nm}$ (Fig. 3A). The sizes of the different proteins were next analyzed by sucrose gradient centrifugation (fig. S3B). The majority of each protein construct migrated as complexes larger than Cytochrome C (25 kDa), but smaller than EHD2 dimers (120 kDa), suggesting that these constructs formed complexes, likely to be trimers. Additionally, small amounts of higher ordered complexes were detected for 
the (1-155), (45-155) and (44-190) protein variants. Our data implied that all the examined Cavin constructs were able to form trimers, which is in line with that the HR1 domain has been shown to form a trimeric $\alpha$-helical coiled coil structure (11).

To study how well the different truncated proteins inserted into membrane, their adsorption to monolayers was monitored. Injection of Cavin1 (1-190) and (1-155) resulted in an immediate steep increase in surface pressure (Fig. 3, B and C). Surprisingly, injection of the proteins lacking the DR1 domain, (44-190) and (44-155), resulted in a considerably lower rate and level of surface pressure increase (Fig. 3, B and $\mathrm{C}$ ). These data show that the presence of the DR1 domain provides a significant effect on the ability of the protein to insert into the monolayer. As the negatively charged DR1 domain does not contain any apparent significant hydrophobic residues that might insert into membranes, we wanted to decipher if it could bind membranes on its own. Cavin1 (1-44) was first expressed and purified from E. coli (fig. S3A) and then injected underneath a monolayer of DOPC:DOPE:PI(4,5)P 2 (fig. S3C). A minimal rise in surface pressure was recorded, indicating that this truncate does not have a high affinity for membranes. Similarly, a 1:1 mixture of DR1 and HR1 domain resulted in a negligible surface pressure increase (fig. S3C). These data suggested that the combined DR1 and HR1 is required for membrane insertion.

\section{DR1 effects the structure of the adsorbed protein to the membrane}

Next, we sought to monitor how conformational changes in the hydrophobic environment would affect membrane binding of Cavin1 (1-155) and (1-190). The two different truncated proteins were analyzed by $\mathrm{CD}$ with or without preincubation with FOLCH liposomes in low salt conditions (Fig. 3D). An increased ratio between the 222 and $208 \mathrm{~nm}$ ellipticities $\left(\left[\theta_{222}\right] /\left[\theta_{208}\right]\right)$ in the $\mathrm{CD}$ spectra can be a sign of an increase in the hydrophobicity of the environment of the helix, which could derive from membrane insertion, or oligomerization, protecting the surfaces from the solvent. In the presence of liposomes, we noticed a dramatic change in the CD spectra of both the 1-155 and 1190 constructs compared to absence of liposomes (Fig. 3D). Both Cavin1 (1-155) and (1-190) showed a significant increase of $-\left[\theta_{222}\right]$ in relation to $-\left[\theta_{208}\right]$, which can be a signature of increasing helical content or that the helices are inserted into a lipid bilayer. This showed that the HR1 domain is affected by membrane binding suggesting that this region might be directly involved in the insertion. 
To further assess if protein insertion was mediated by the DR1-HR1 domain influenced membrane association, we assayed the adsorption of Cavin1 (1-190) and (44-190), which lacks the DR1 domain, to SLBs using QCM-D. Both constructs demonstrated a strong adsorption to the SLB in buffer with $150 \mathrm{mM} \mathrm{NaCl}$ (Fig. 3, E and F, grey area); however, there was a significant difference between the constructs when the SLB was rinsed with the same buffer (Fig. 3, E and F, white area). While the Cavin1 (1-190) showed a slow release during the buffer rinse similar to the full-length protein (Fig. 1D), a large fraction of Cavin1 (44-190) (58 $\pm 10 \%)$ was immediately released. This indicated that (1-190) was more stably attached to the surface as compared to Cavin1 (44-190) (Fig. 3, E and F). In agreement with this, the adsorption of (44-190) resulted in a much larger increase in dissipation than (1-190), suggesting that the adsorbed (44190) protein is much less rigid than the (1-190). To facilitate easier interpretation of the data "softness plots", which decouple the relationship between the frequency and dissipation, were created (Fig. 3G). In these plots values closer to zero represent greater rigidity, while larger values represent a greater softness of the surface. Additionally, arrows were added to indicate the direction of time. We found that adsorption of both proteins made the surfaces more rigid but that (44-190) (magenta line) created a much softer layer on the surface than (1-190) (Fig. 3G, black line). Rinsing the system induced desorption of both proteins. Desorption of the (1-190) protein made the surface softer in a reverse manner as to that seen for the adsorption (Fig. 3G, grey line). Interestingly, the surface became even more rigid when Cavin1 (44-190) desorbed (Fig. $3 \mathrm{G}$, pink line).

\section{Cavin1 (1-190) adsorbs slightly inclined to membranes as determined by IRRAS}

To address how the HR1 domain in combination with the DR1 and DR2 domains would orient at the membrane, we used IRRAS to get direct information on the secondary structure of the adsorbed protein and its orientation (23). The secondary structure of the protein is encoded in the position of the amid I' band whereas the orientation influences its intensity. Knowledge of the protein structure is essential for appropriate data analysis and determination of the orientation at the lipid monolayer. Since the crystal structure of the HR1 (45-155) is known (11) and both DR1 (1-44) and DR2 (156-190) lack a secondary structure, we could use this technique to understand the membrane association of Cavin1 (1-190) in more detail. The asymmetric amid I' band shape in the 
experimental spectra indeed indicated that in addition to helical structures, further components such as unordered structural elements were present, Moreover, the positions of the helical components are typical for a coiled coil structure (38) This is in agreement with the combination of HR and DR domains (11). To predict orientation of Cavin1 (1-190) when adsorbed to a DOPC:DOPE:PI(4,5) $\mathrm{P}_{2}$ monolayer, experimental and simulated IRRA spectra recorded at various angles of incidence with p- or spolarized IR-light were compared (Fig. 4A). The best fitting band simulation yielded an average inclination angle of $\gamma=22.5 \pm 2.5^{\circ}$ for the helical components of Cavin1 (1190) with respect to the lipid monolayers (Fig. 4A). However, when adsorbed to $\mathrm{PI}(4,5) \mathrm{P}_{2}$ containing lipid monolayers (Fig. 4A). However, upon fitting simulated to experimental spectra at all theoretically possible inclination angles, we found that the minimum is rather shallow and spectral fits are reasonable for average helix inclination angles between $0^{\circ}$ (parallel to the lipid layer, fig. S4A) and $30^{\circ}$ (slightly inclined, Fig. 4B). Conversely, at higher inclination angels $\left(\gamma>30^{\circ}\right)$ no acceptable spectral fit could be obtained (fig. S4B). It should be noted that an inclination angle of $\gamma=0^{\circ}$ could only be obtained if the HR1 helix bundle dissociates and all three helices adsorb individually to the interface. In case of HR1 adsorption as intact trimer, the smallest possible helix inclination angle, would be $\gamma=12^{\circ}$ due to the intrinsic helix orientations within the bundle. The minimum found in the IRRA spectra fit corresponds to a HR1 bundle inclination of $20 \pm 3^{\circ}$ (Fig 4C). Our results implied that the HR1 domain of Cavin1 (1190) adsorbs with a slight inclination to the interface, where average inclination angles of $17-23^{\circ}$ were most probable (Fig. 4C). While our analysis showed that a steeper orientation of the helices was unfeasible, a flatter, almost parallel orientation seemed possible, especially if protruding or dangling uncoiled segments would be present.

\section{Binding and orientation of the HR1 domain at the membrane interface.}

Next, we aimed to mechanistically dissect how the DR1-HR1 domain would bind and insert into membranes using molecular dynamics (MD) simulations. We limited the computational model to the HR1 domain, since the structure is described in detail, and it is the part of the protein known to interact with lipids. To address membrane binding of the HR1 domain, we performed coarse-grained MD (CG-MD) simulations, where the HR1 domain was placed in a simulation box near a lipid membrane composed of 
DOPC:DOPE:PI $(4,5) \mathrm{P}_{2}$ to monitor protein-membrane interactions over a time-frame of $2 \mu \mathrm{s}$ (Fig. 5A). The initial binding of the HR1 trimer to the membrane occurred at an approximate angle of $56 \pm 7^{\circ}$. Comparison to membranes composed of only DOPC/DOPE, where no binding occurred (fig. S5A), revealed that the presence of $\mathrm{PI}(4,5) \mathrm{P}_{2}$ in the membrane was essential for binding. We found that lysine residues in either the $\mathrm{N}$ - or C-terminus of the HR1 domain initiated the binding to $\mathrm{PI}(4,5) \mathrm{P}_{2}$ at a probability of $33 \%$ and $67 \%$, respectively (Fig. 5A). Subsequently, interactions between $\mathrm{PI}(4,5) \mathrm{P}_{2}$ and positively charged residues along the trimer surface resulted in a horizontal binding of the HR1 in relation to the membrane in all simulations (Fig. 5A and fig. S5B), which is in agreement with the IRRAS data. In this state, the HR1 was tightly packed towards the head group interphase. However, the individual helices facing the membrane appeared to be different in between simulations (fig. S5B). To decipher if the trimer orientation changed once bound in the horizontal state, we calculated the rotation angle $\theta$ of the HR1 domain over the membrane plane during the simulation. The rotation angle profile indicated that $\theta$ varied only before horizontal binding of the HR1 domain to the membrane (fig. S5C). Once horizontally bound, the orientation of the HR1 did not change during the simulation (the $\theta$ value remains close to zero), suggesting that the random initial binding of a particular residue in one of the helices determined the orientation at the membrane interface.

In addition, we performed all-atom MD simulations as these provide more detailed models at higher accuracy and allow studying hydrogen bond formation between proteins and lipids (Fig. 5B). In these simulations, the trimer was placed close to the membrane surface, before reaching the surface within $10 \mathrm{~ns}$ and instantly attaching to it. We observed that all three helices were engaged in hydrogen bonding once the trimer was horizontally attached and that $68 \%$ of the bonds were formed between the trimer and $\mathrm{PI}(4,5) \mathrm{P}_{2}$ (fig. S5D). Especially residues in both the $\mathrm{N}$ - or $\mathrm{C}$-terminal region of the HR1 domain contributed to most of the hydrogen-bonding interactions. This was confirmed by the solvent accessible surface area (SASA); a measure of whether residues in the protein are buried or solvent exposed. The SASA is visualized in yellow in Figure 5B, indicating a dense packing of HR1 and membrane as seen in the GC simulations. 
Next, we analyzed whether a particular orientation of the trimeric HR1 domain towards the membrane is would be energetically favorable. For this, we simulated the rotation of the trimeric HR1 domain around its helical axis and evaluated the free energy of protein-membrane interactions by removing the protein from the simulation box at different rotation angles (Fig. 5, C and D). This enabled us to compare at which orientation the protein was more stable, indicated by a lower energy state of the system. Thermodynamic integration revealed alteration of the free energy as the trimer underwent gradual rotation around its helical axis (Fig. 5D). However, the difference between the lowest and the highest energy states was found to be of approximately $3 \%$ of the entire protein decoupling energy. This is in line with the CG simulations, suggesting that the HR1 domain will bind to the membrane in any of these most energetically favorable orientations although the precise helices engaged in binding can vary. In line with this, the positively charged residues interacting with $\mathrm{PI}(4,5) \mathrm{P}_{2}$ are distributed homogeneously around the surface of the rod-like HR1 domain (Fig. 5E). This is in contrast to previously described membrane binding domains such as BAR domains, which have a preferred membrane interaction surface. This shows that the HR1 domain is distinct from previously characterized membrane interacting domains and that this novel type of membrane binding domain is designed to engage with the membrane in a distinct way.

To determine the position of the HR1 at the bilayer we used CG-MD simulations (Fig. 3B). On average the distance between the head groups and the center of the bilayer was $1.95 \mathrm{~nm}$ (29). The residues of HR1 closest to the membrane were on average $1.79 \pm$ $0.15 \mathrm{~nm}$ from the bilayer center suggesting that the HR1 was shallowly buried in the membrane in between the head groups. This could account for the intermediate increase in surface pressure detected for the HR1 domain in the monolayer experiments (Fig. 3B). Interestingly, the CG-MD simulation also showed that the helices in the HR1 could become slightly uncoiled (fig. S5B, simulation 6), suggesting that membrane interaction may induce partial separation of the helices in the HR1 trimer (Fig. 5B). When binding of single chain helices to the membrane was simulated by using CG-MD (Fig. 5F), we observed that the residues of the individual helices were inserted into the membrane on average $1.42 \pm 0.25 \mathrm{~nm}$ from the bilayer center. This suggested that partial uncoiling of the trimer would allow for the helices to insert deeper into the membrane (below the head group region) compared to the HR1 trimer. In summary, 
our data suggested that the HR1 was engaged in initial electrostatic membrane binding, but that the DR1 domain affected the orientation and potential partial separation of the helices in HR1 to facilitate insertion into the membrane leaflet.

\section{Membrane insertion of cavin1 influence caveolae coat stability and co-assembly with CAV1 in cells}

To analyze if the described membrane insertion of Cavin1 influenced the stability of the caveolae-coat, we purified caveolae enriched membrane (CEM) fractions from mouse lung tissue as previously described (30). The CEM was subsequently subjected to either $4 \mathrm{M} \mathrm{NaCl}$ (to compete with electrostatic interactions), methyl- $\beta$-cyclodextrin ( $\mathrm{M} \beta \mathrm{CD}$; to remove cholesterol) or octylglucoside (oct gluc; to completely dissolve the membranes) treatment before flotation using a sucrose step gradient centrifugation to collect the CEM fraction. Immunoblotting of the CEM fractions revealed that CAV1, Cavin1, 2 and 3 and EHD2 were present in the control (untreated) sample (Fig. 6A). However, removal of cholesterol or octylglucoside treatment dissolved the caveolae in accordance with previous studies (8). Interestingly, $\mathrm{NaCl}$ treatment increased the association of Cavin1, 2 and 3 with the CEM-fraction but reduced EHD2 association (Fig. 6A). High ionic strength would reduce electrostatic (ionic) interactions and correspondingly, enhance hydrophobic interactions, suggesting that hydrophobic interactions indeed play a central role for the stability of the caveolae-association of the Cavins.

Our data suggested that the N-terminal DR1 domain of Cavin1 is instrumental to enforce membrane insertion of the protein. To test how removal of the DR1 domain would affect association with caveolae in vivo, we expressed fluorescently tagged CAV1 together with either the full length and $\triangle \mathrm{DR} 1$ (44-392) constructs in PC-3 cells. These cells lack endogenous Cavin1 and therefore also caveolae as CAV1 is dispersed in the plasma membrane (fig. S6A) $(18,31)$. Following expressions of Cavin1-GFP, typical punctate CAV1-RFP and Cavin1-GFP-positive structures indicative of caveolae were detected at the plasma membrane using confocal microscopy (Fig. 6B). However, expression of Cavin1- $\triangle \mathrm{DR} 1$ resulted in the majority of CAV1 being present in larger internal membranous structures devoid of Cavin1- $\triangle \mathrm{DR} 1-\mathrm{GFP}$ (Fig. 6B, arrows). This CAV1 phenotype is similar to the phenotype described by Tillu et al. (18). At the plasma membrane, we detected CAV1-positive structures that were both positive and 
negative for $\triangle \mathrm{DR} 1$, suggesting that part of the Cavin $1-\triangle \mathrm{DR} 1$ could assemble together with CAV1. In agreement with previous work (18), we observed that the $\triangle \mathrm{DR} 1$ construct did localize to caveolae similarly to full length Cavin1 in HeLa cells, which have endogenous Cavin1. This suggests that the $\triangle \mathrm{DR} 1$ can assemble on caveolae given that endogenous Cavin1 is present (fig. S6B). Taken together, these data indicate that the DR1-mediated insertion of Cavin1 influence the co-assembly together with CAV1. 


\section{DISCUSSION}

The caveola-coat has eluded detailed architectural description for decades, but based on structural analysis of both caveolae and the components building up the coat, different models have been proposed $(13,14,32)$. Validation and further improvements of these models rely on detailed structural understanding of the individual protein components at the membrane interface. In this work, we have studied the initial binding and assembly of Cavin1 at the membrane interface. We found that Cavin1 inserted into the membrane via a mechanism dependent on the DR1-HR1 interacting unit, which we propose to undergo dynamic transitions between a shielded coiled coil state in solution and a partially uncoiled state, which is partially buried in the membrane. Helix insertion has been shown to drive membrane curvature (33), but also to provide lipid specificity and targeting to specific cellular compartment as in the case of proteins containing amphipathic lipid packing sensor (ALPS) motifs (34). The described membrane insertion of Cavin1 would allow it to directly interact with lipid acyl chains. Such interactions could be linked to the recently reported lipid sorting activity of the caveolae coat, which was shown to be sensitive to the saturation level of acyl chains (5). The proposed mechanistic steps could provide specificity and regulation to the assembly process and enable Cavin1 to generate membrane curvature and interact with the integral part of CAV1 below the head group region of the membrane.

To characterize the properties of Cavin 1 membrane binding, we used purified full length and truncated versions of Cavin1 in combination with different membrane model systems. Whereby, we have been able to reconstitute and measure membrane binding and insertion of Cavin1. The use of supported lipid bilayers in combination with QCMD provides a valuable tool for tracking and quantification of membrane binding and protein release in real time as well as a means to probe the role of electrostatic interactions in these processes. By using this methodology, we could demonstrate that Cavin 1 rapidly adsorbed to the membrane, followed by a subsequent slow salt-resistant release of the protein. We also found that the change in membrane stiffness caused by Cavin1 (1-190) binding was reversible and proportional to the amount of bound protein. This suggested that the protein adsorption was organized and that the effects on membrane properties were directly coupled to the bound protein. In comparison, deletion of DR1 resulted in a slightly increased rate of protein adsorption, but the 
release of the protein was much more rapid and the effects on the membrane stiffness were not directly proportional to the amount of bound protein. This indicated that deletion of the DR1 resulted in less organized binding involving different bound states.

Surface pressure measurements using Langmuir lipid monolayers, showed that Cavin1 rapidly inserts into lipid monolayers containing $\mathrm{PI}(4,5) \mathrm{P}_{2}$. This was indicated by a steep increase in surface pressure, showing that the protein inserted in between the head groups of the lipids. The MIP of cavin1 was higher than the monolayer-bilayer equivalence pressure, suggesting that Cavin1 spontaneously inserts into cellular membranes. The MIP is furthermore very similar to other proteins shown to insert into membranes using this methodology such as the BAR-domain of Bin1 (35) and Sar1p (36), Interestingly, the MIP of Cavin1 (1-190) was much higher than the full length protein. This might suggest that the $\mathrm{N}$-terminal region is restrained by the $\mathrm{C}$-terminal region in the full-length protein, and that the helical region might more easily uncoil to mediate membrane insertion following truncation. Insertion was dependent on the combined DR1 and HR1 regions, but neither of these were able to insert on their own, suggesting that the interplay between the negatively charged DR1 and the positively charged HR1 is important. With IRRAS we showed that monolayer adsorption insertion of the N-terminal part of Cavin1 was dependent on $\mathrm{PI}(4,5) \mathrm{P}_{2}$ in the monolayer, in agreement with previous results $(18,19)$. The data also showed that the adsorbed protein was inserted below the head group region of the lipids, supporting the pressure measurements. We found that the presence of Cavin1 (1-190) influenced the membrane organization, which is a prerequisite for membrane remodeling. Comparison of the IRRA spectra to the theoretical spectra of the HR1 based on the crystal structure revealed that the HR1 region was bound to the membrane in a slightly inclined orientation in relation to the membrane.

Using a combination of CG and all-atom MD simulations, we were able to dissect the binding interface of the HR1 and the hydrogen bonds formed with $\mathrm{PI}(4,5) \mathrm{P}_{2}$. We found that binding was initiated and further supported by electrostatic interactions between $\mathrm{PI}(4,5) \mathrm{P}_{2}$ and lysine residues along HR1 region resulting in tight horizontal docking of the HR1 domain. This is similar to pervious simulations with a different lipids composition, POPC:POPS:PI(4,5) $\mathrm{P}_{2}$ (80:15:5 mol\%) (5). Interestingly, we found that different helices of the trimeric HR1 were facing the membrane in the individual 
simulations. Careful analysis of the energies coupled to the orientation of the membrane bound HR1, revealed that the differences in $\Delta \mathrm{G}$ following rotation of the HR1 were very small in comparison to the overall binding energy of the HR1. This is in agreement with the fact that the positively charged residues are distributed homogeneously around the surface of the rod-like HR1 trimer, showing that HR1 can bind in either of these orientations. Furthermore, we noticed that the coiled coil in the N-terminus of HR1 trimer appeared to be slightly uncoiled (Fig. 5B), and that individual helices of the HR1 were able to insert deeper into the membrane as compared to the trimer. In addition, analysis of the CD spectra suggested that conformational changes take place in the HR1 when bound to liposomes (Fig. 3D). Taken together these results support the idea that the HR1 trimer might partially uncoil to expose hydrophobic residues, hidden inside the coiled core, during membrane binding and insertion. Helical uncoiling could be driven by the interactions between $\mathrm{PI}(4,5) \mathrm{P}_{2}$ headgroups and the individual helices in a rotational movement competing with the interactions stabilizing the coiled coil. Similar drastic conformational changes have been observed in other proteins upon membrane binding. For example, membrane-driven exposure of amphipathic helixes in small Gproteins (33) and the major helical rearrangement in pore forming proteins Bak and Bax upon membrane binding (37).

Here, we identified the DR1-HR1 unit to be responsible for initial membrane binding and insertion. However, previous data has shown that the HR2 region can interact with PS lipids via the undecad region provided that the HR1 region is present (17). This indicates that the whole protein might be engaged in the membrane adsorption process. In line with this, the disordered regions were proposed to influence assembly of Cavin1 via "fuzzy" electrostatic interactions with the helical regions. This induces a liquidliquid phase separation and contributes to molecular crowding, which was proposed to drive membrane curvature and generate a meta-stable caveolae coat (18). Our in vitro and in silico data are consistent with this model and extend the implications of the weak electrostatic interactions between the DR1 and HR1 to membrane insertion. The precise role of the DR1 is not clear, but could involve stabilization of a partially uncoiled state of the HR1 to support membrane insertion as indicated by our data. Post-translational modifications might also influence membrane binding. In our mass spectrometry analysis of Cavin 1 purified from mammalian cells, we found that the disordered regions were heavily phosphorylated. This is in line, with previous data (19), and would 
strengthen the negative charge of these regions and potentially their role in electrostatic interactions. In addition, we discovered a novel serine ubiquitination site near the DR3 region. This region has been shown to be important for the interaction of Cavin1 with CAV1 (18), but the role of the ubiquitination has to await further studies.

Our findings also raise the question whether membrane insertion is universal to all four Cavin species. The Cavins share the same domain organization with alternating disordered and helical regions, which display high sequence similarity (11). Previous studies showed that Cavins homo- and hetero-oligomerize via HR1 domain, and that the N-terminal part of the HR1 (amino acids 44-100) of Cavin1 is necessary and sufficient for trimerization (12). The C-terminal part of HR1 in Cavin3 is less homologous, where bulky residues have been substituted by alanine, which may result in reduced stability of the coiled coil. The hetero-oligomerization of Cavin1 with either Cavin 2 or 3 could hereby potentially influence the stability and uncoiling of the Cterminal part of the HR1. However, it remains to be addressed if the other Cavins insert into membranes via a similar mechanism.

In agreement with previous studies (18), we found that removal of the DR1 region did not majorly affect the localization of Cavin1 to caveolae in HeLa cells where the endogenous Cavin1 was present. However, expression of a Cavin1- $\Delta \mathrm{DR} 1$ mutant in PC-3 cells, which lack endogenous Cavin1, showed that this construct was not able to assemble CAV1 in caveolae the same way as the wild type protein. Instead, CAV1 was detected in large intracellular structures, reminiscent of endosomal structures enriched in CAV1. This CAV1 phenotype is similar to the phenotype described by Tillu et al. (18). Yet, in our study, we did not observe Cavin1- $D$ DR1 on the large CAV1-positive intracellular structures, which might be due to the different experimental setup where we analysed live cells expressing CAV1-RFP. Taken together, the dynamic membrane insertion mechanism of Cavin1 described here provides a mechanistic basis for membrane-assisted regulation of the caveolae coat assembly. 


\section{MATERIALS AND METHODS}

\section{Lipids}

POPC (1-palmitoyl-2-oleoyl-glycero-3-phosphocholine), $\quad \mathrm{PI}(4,5) \mathrm{P}_{2} \quad(\mathrm{~L}-\alpha-$ phosphatidylinositol-4,5-bisphosphate, porcine brain, ammonium salt), DOPC (1,2dioleoyl-sn-glycero-3-phosphocholine), DOPE (1,2-dioleoyl-sn-glycero-3phosphoethanolamine) and brain total lipid extract (FOLCH fraction, porcine) were purchased as lyophilized powder from Avanti Polar Lipids, Inc. (Alabaster, AL, USA).

\section{Protein purification}

Cavin1 truncation proteins: 1-44, 44-155, 44-190, 1-155, 1-190 and 191-392, were purified as described previously (12). Proteins were expressed with $\mathrm{N}$-terminal $6 \times \mathrm{His}-$ tags in E. coli Rosetta pLysS or BL21(DE3)pLysS (growth in Terrific Broth media). Protein expression was induced with $1.0 \mathrm{mM}$ IPTG at the exponential phase and incubated overnight at $20^{\circ} \mathrm{C}$. TALON Superflow (Cytiva, Uppsala, Sweden) was used for affinity purification. Imidazole was removed either by gel filtration chromatography using Sephacryl S-300 HR (Cytiva) or spin columns Bio-Spin (Bio-Rad, Hercules, USA) exchange of protein in $20 \mathrm{mM}$ HEPES buffer (pH 7.4 containing $300 \mathrm{mM} \mathrm{NaCl}$ ). Full length Cavin1 and $\triangle \mathrm{DR} 1$ mutants were expressed and purified from suspension cells HEK-293F (Invitrogen, Carlsbad, CA, USA) as described previously (38). Cells were grown to $2-3 \times 10^{6}$ cells $\mathrm{ml}^{-1}$ on a shaker $\left(160 \mathrm{rpm}\right.$.) at $37^{\circ} \mathrm{C}$ with $8 \% \mathrm{CO}_{2}$ in 4 $\mathrm{mM}$ glutamine supplemented BalanCD medium (Irvine Scientific, Wicklow, Ireland). A total of $1 \mu \mathrm{g}$ per one million cells of the plasmids containing CMV promoter and $3 \times$ FLAG-tagged genes, and were mixed with a threefold excess (w/w) of polyethylenimine MAX $40 \mathrm{kDa}$ (Polysciences, Warrington, PA, USA) in $4 \mathrm{ml}$ OptiPro (Invitrogen). The mixture was incubated for $20 \mathrm{~min}$ at room temperature before being added to the cell cultures. Cells were grown for two days with an addition of 5\% BalanCD Feed (Irvine Scientific) per day. Cells were then centrifuged at $350 \mathrm{~g}$ for 5 min, and lysed with 1\% Nonidet P40 (Thermo Fisher Scientific, Waltham, MA, USA) for $15 \mathrm{~min}$ on ice. Following centrifugation ( $350 \mathrm{~g}, 5 \mathrm{~min}$ ), the supernatant was stored at $-80^{\circ} \mathrm{C}$ for further purification. The lysate was thawed and centrifuged at 20,000 $g$ for $10 \mathrm{~min}$ and the supernatant was added to $3 \mathrm{ml}$ anti-Flag (M2)-agarose (Sigma, St. Louis, MO, USA), and incubated at $4^{\circ} \mathrm{C}$ overnight. The gel matrix was transferred to a column 
and washed with 10 column volumes of $20 \mathrm{mM}$ HEPES, $300 \mathrm{mM} \mathrm{NaCl}$ (pH 7.4). In order to remove Hsp70 chaperones, the matrix was incubated with a buffer containing $5 \mathrm{mM}$ ATP, $20 \mathrm{mM} \mathrm{MgCl}_{2}, 10 \mathrm{mM} \mathrm{KCl}$ and $0.1 \%$ Nonidet $\mathrm{P} 40$ for 2 hours. The protein was eluted with $100 \mu \mathrm{g} / \mathrm{ml}$ of $3 \times$ FLAG peptide (Sigma). The eluted protein was adjusted to desired concentration via Vivaspin (Sartorius, Göttingen, Germany), analyzed by SDS-PAGE and snap frozen in liquid nitrogen, and stored at $-80^{\circ} \mathrm{C}$.

\section{Tandem mass spectrometry}

Proteomic analysis was performed at The Proteomics Core Facility at the Sahlgrenska Academy, University of Gothenburg, Sweden. After electrophoresis and coomassie staining, the protein band of full-length Cavin1 was cut-out and submitted to the facility. The gel band was destained with $25 \mathrm{mM}$ ammonium bicarbonate in $50 \%$ acetonitrile (ACN), in-gel digested by addition of $10 \mathrm{ng} / \mu \mathrm{l}$ trypsin (Pierce MS grade, Thermo Fisher Scientific) in $50 \mathrm{mM}$ ammonium bicarbonate and incubated overnight at $37^{\circ} \mathrm{C}$. Peptides were extracted from the gel for $20 \mathrm{~min}$ in $75 \% \mathrm{ACN}$ in $0.2 \% \mathrm{TFA}$ followed by $50 \% \mathrm{ACN}$ in in $0.2 \%$ TFA. The extracted peptides were dried by vacuum centrifugation and reconstituted in $15 \mu \mathrm{l}$ of $0.1 \% \mathrm{FA}$ (formic acid) in $3 \% \mathrm{ACN}$. The peptide sample was analyzed on an Orbitrap Fusion Lumos Tribrid mass spectrometer interfaced with Easy-nLC1200 liquid chromatography system (Thermo Fisher Scientific). Peptides were trapped on an Acclaim Pepmap 100 C18 trap column (100 $\mu \mathrm{m} \times 2 \mathrm{~cm}$, particle size $5 \mu \mathrm{m}$, Thermo Fisher Scientific) and separated on an in-house packed analytical column $(75 \mu \mathrm{m} \times 35 \mathrm{~cm}$, particle size $3 \mu \mathrm{m}$, Reprosil-Pur C18, Dr. Maisch) using a linear gradient from 5\% to $35 \%$ B over 47 min followed by an increase to $100 \% \mathrm{~B}$ for $3 \mathrm{~min}$, and $100 \% \mathrm{~B}$ for $10 \mathrm{~min}$ at a flow of $300 \mathrm{nl} / \mathrm{min}$. Solvent A was $0.2 \%$ formic acid and solvent B was $80 \%$ acetonitrile, $0.2 \%$ formic acid The mass spectrometer was operating in data-dependent mode with $3 \mathrm{~s}$ maximal duty cycle, with the precursor ion spectra followed by the HCD spectra at the collision energy of $30 \%$ with the dynamic exclusion duration for 45 s. Protein identification was performed using Proteome Discoverer version 2.4 (Thermo Fisher Scientific). The LC-MS data was matched against the Mouse Musculus Uniprot database from July 2020, using Mascot 2.5.1 (Matrix Science, London, United Kingdom) as a search engine with trypsin and 1 allowed missed cleavage as an enzyme rule, with the precursor tolerance of $5 \mathrm{ppm}$ and fragment tolerance of $0.7 \mathrm{Da} ; \mathrm{M}$ oxidation, S/T/Y phosphorylation, $\mathrm{K}$ 
gluratylation, GluGlu addition on E/S/T/S was set as variable modifications, and C methylthiolation was set as a fixed modification. Fixed Value PSM validator was used to assess the quality of peptide matches with False Discovery rate of 0.01 .

\section{Liposome co-sedimentation assay}

The liposome co-sedimentation assay was performed according to a previously published method (39). Briefly, FOLCH lipids or formulated lipid mixtures composed of DOPC:DOPE:PI(4,5)P 2 (55:45:5 mol\%) were dissolved to a final concentration of 1 $\mathrm{mg} / \mathrm{ml}$ in chloroform: methanol $(3: 1 \mathrm{v} / \mathrm{v})$. Lipids were dried under a stream of nitrogen, and resuspended in $20 \mathrm{mM}$ HEPES buffer, $150 \mathrm{mM} \mathrm{NaCl}(\mathrm{pH} 7.4)$ in a bath sonicator (Transsonic T310, Elma Schmidbauer, Singen, Germany). Proteins were incubated with liposomes at a final concentration of $3 \mu \mathrm{M}$ and $0.5 \mathrm{mg} / \mathrm{ml}$, respectively, for $15 \mathrm{~min}$ at room temperature. The samples were centrifuged at 100,000 $\mathrm{g}$ for $20 \mathrm{~min}$ at room temperature. Then the supernatant and pellet were analyzed by Coomassie stained SDSPAGE, and quantified using Image Lab software (Bio-Rad).

\section{Supported lipid bilayer (SLB) and quartz crystal microbalance with dissipation monitoring (QCM-D)}

Vesicles for foaming SLBs were prepared as described in liposome co-sedimentation assay. Except for the vesicles containing POPC:PI(4,5) $\mathrm{P}_{2}(95: 5 \mathrm{~mol} \%)$, which were extruded 11 times (Extruder, Avanti). through a polycarbonate filter (Nuclepore TrackEtched Membranes, Whatman, Maidstone, UK) with $100 \mathrm{~nm}$ pore size An AWSensors X4 unit (AWSensors, Valencia, Spain) equipped with a flow chamber was used to conduct the QCM-D measurements. Wrapped $14 \mathrm{~mm}\left(5 \mathrm{MHz}, \mathrm{Cr} / \mathrm{Au}-\mathrm{SiO}_{2}\right.$, polished) sensors were used for all experiments. Each sensor was stored in $2 \%$ sodium dodecyl sulfate (SDS) overnight and treated with UV-ozone (Bioforce Nanosciences, USA) for 30 min prior to use. The frequency and dissipation changes for overtones 1, 3, 5, 7, 9, and 11 were all recorded, but only the third overtone was reported herein. POPC:PI(4,5)P 2 vesicles $(100 \mu \mathrm{l}, 0.1 \mathrm{mg} / \mathrm{ml})$ in $20 \mathrm{mM}$ citrate, $50 \mathrm{mM} \mathrm{KCl}, 0.1 \mathrm{mM}$ EDTA ( $\mathrm{pH} 4.5$ ) were injected in a continuous flow and SLB formation was monitored. After SLB formation, the chambers were rinsed with the buffer (20 mM HEPES, 150 $\mathrm{mM} \mathrm{NaCl}, \mathrm{pH}$ 7.4). After reaching a stable baseline, protein was injected into the chamber. Flow was paused once the protein solution had filled the sensor chamber and the system was allowed to reach equilibrium, before rinsing with the buffer. High salt 
treatment was done by rinsing the sensor surface with the same buffer but a higher salt concentration $(300 \mathrm{mM} \mathrm{NaCl})$ followed by rinsing with the initial buffer $(150 \mathrm{mM}$ $\mathrm{NaCl})$.

\section{Lipid monolayer experiments}

Lipid monolayer experiments were performed with either a custom-built round PTFE trough at Martin-Luther-Universität, Halle-Wittenberg $(\varnothing 60 \mathrm{~mm} \times 3 \mathrm{~mm}$, Riegler and Kirstein GmbH, Potsdam, Germany) or a Microtrough G1 system at Umeå University (Ø $53 \mathrm{~mm} \times 4 \mathrm{~mm}$, Kibron, Helsinki, Finland). Both troughs were covered to prevent temperature and humidity loss and temperature of the subphase was controlled through a circulating water bath. Lipid mixtures consisting of DOPC:DOPE:PI(4,5) $\mathrm{P}_{2}$ (55:45:5 mol\%) were prepared at total lipid concentration of $1 \mathrm{mM}$ in chloroform:methanol (3:1 $\mathrm{v} / \mathrm{v})$. A microbalance equipped with a Wilhelmy plate was used to measure the surface pressure $(\pi)$ and calibrated before each measurement. Lipid solutions were deposited onto the surface of the subphase (25 mM HEPES, $300 \mathrm{mM} \mathrm{NaCl}, 25 \mathrm{mM} \mathrm{KOH}$, pH 7.4) to obtain the required initial surface pressure $\pi_{0}$. The subphase was continuously stirred by magnetic stirrer After the solvent had been allowed to evaporate for $15 \mathrm{~min}$ and a stable monolayer was formed, the protein was injected under the lipid film directly into the subphase using a thin syringe needle (final concentration $50 \mathrm{nM}$ ). Curve analysis of $\Delta \pi / \pi_{0}$ plot provides synergy factor $(a)$ as the slope of the linear regression $>1$. A positive $a$ is indicative for attractive interactions between the lipid monolayer and the injected protein, while $a=0$ would indicate a lack of interactions. The maximum insertion pressure (MIP) was determined from the $\Delta \pi / \pi_{0}$ plot through linear extrapolation to the x-axis, i.e., it corresponds to $\pi_{0}$ at $\Delta \pi=0$ (25). The standard deviation of the MIP value was calculated according to the formula given in (25). Analysis of the adsorption curves was performed with Origin 8.1 (Origin Lab Corp., Northampton, MA, USA).

\section{Monolayer measurements with infrared reflection-absorption spectroscopy (IRRAS)}

The Langmuir trough system used in combination with IRRAS (Riegler and Kirstein $\mathrm{GmbH})$ included a circular sample $(\varnothing 60 \mathrm{~mm} ; 7.4 \mathrm{ml})$ and a rectangular reference trough $(30 \times 6 \mathrm{~cm})$. The levels of the subphase (either $\mathrm{H}_{2} \mathrm{O}$ or $\mathrm{D}_{2} \mathrm{O}$ based) were 
controlled with an in-built laser and could be externally regulated via a pump system. The subphase was maintained at $20^{\circ} \mathrm{C}$ through a circulating water bath. The same procedure as above was followed by preparation of the lipid film. IRRAS experiments were conducted with a Bruker Vertex 70 FTIR spectrometer equipped with an A 511 reflection unit (Bruker, Karlsruhe, Germany) and an external mercury cadmium telluride (MCT) detector. The entire setup was enclosed and purged to keep the relative humidity constant. IRRA spectra of the films were acquired at various angles of incidence (between 25 and $70^{\circ}$ ) using parallel (p) and perpendicularly (s) polarized infrared light. 2000 scans were accumulated in $\mathrm{p}$ and 1000 in s polarization of the IR beam with a resolution of $8 \mathrm{~cm}^{-1}$ and a scanner frequency of $80 \mathrm{kHz}$. An additional zero filling factor of 2 was applied to the averaged interferograms prior to Fourier transformation. The single-beam reflectance spectra of the reference $\left(\mathrm{R}_{0}\right)$ and the sample $(\mathrm{R})$ trough surfaces were used to calculate the reflection absorption spectrum as $\lg \left(\mathrm{R} / \mathrm{R}_{0}\right)$.

IRRA spectra of the protein containing monolayer recorded on a $\mathrm{D}_{2} \mathrm{O}$ subphase (Sigma) were simulated in the range of the amid I' vibration according to a three-layer model reported by Kuzmin et al.(40). Simulation and fitting of the multicomponent IRRA bands were performed as described in Schwieger et al. (41). The optical constant of the subphase $\mathrm{D}_{2} \mathrm{O}$ were taken from Berti et al. (42). The refractive index of the lipid/polymer film was set to 1.41 and its layer thickness to $2 \mathrm{~nm}$. The band positions of the subcomponents were derived from second derivative spectra, revealed the contribution of three amide I' band components centered at 1662, 1642, and $1626 \mathrm{~cm}^{-}$ 1. These components were assigned to one random and two $\alpha$-helical secondary structure elements, respectively (39). According to literature, the lowest component could also be related to $\beta$-sheet amid I' vibrations (43). However, neither the X-ray structure nor $\mathrm{CD}$ spectra gave any indication for the presence of $\beta$-sheet components in the structure of Cavin1. Rather, we assigned the two lower spectral components to water exposed and water shielded faces of the helices, respectively, as reported for other coiled-coil helical arrangements (28). In analogy, we assumed that the amide bonds in helical structures facing the aqueous subphase are more water accessible and absorb at lower wavenumbers $\left(1626 \mathrm{~cm}^{-1}\right)$, whereas the interaction with lipid monolayer reduces hydrogen bonds with hydration water leading to a shift to higher wavenumbers (1642 $\mathrm{cm}^{-1}$ ). The band component assigned to amide I' vibrations of unordered structures 
$\left(1662 \mathrm{~cm}^{-1}\right)$ was assumed to originate from isotropically distributed amid bonds and, therefore, simulated with an order parameter of $S=0$ (corresponding to a tilt angle $\theta$ of $54^{\circ}$ with respect to the interface normal). The polar angle between the helix main axis and the transition dipole moments of the helix amid I' vibrations was set to $\alpha=38^{\circ}$. The main axis tilt angle of the two lower band components were set to be identical and varied in a least square Levenberg-Marquardt fit to determine the most probable helix orientation. Further fitting parameters were the full-widths at half-height (fwhh) of the three band components as well as the respective absorption coefficients $k$ max. The spectra were fitted in the range of $1610-1670 \mathrm{~cm}^{-1}$ and at angles of incidence, $\varphi=30$ $70^{\circ}$ in increments of $4^{\circ}$, where $\varphi=49,52,55$ and $58^{\circ}$ were omitted from the fit, because of low reflectivity in the range of the Brewster angle. For better understanding and coherence with the MD simulation results the determined tilt angles $\theta$, which are defined with respect to the interface normal are translated into inclination angles $\gamma$, which are defined in relation to the plane of the interface $\left(\gamma=90^{\circ}-\theta\right)$. The confidence interval of the fit minimum was calculated as follows: the goodness of the fit was assessed as the sum of square deviations at each inclination angle $\gamma$ (SSD). These values were weighted by their minimum with ( $\mathrm{N}-\mathrm{p})$, were $\mathrm{N}=360$ is the number of fitted data points and $p=6$ is the number of fitted parameters:

$$
X=\frac{S S D-S S D_{\min }}{S S D_{\min }}-1 \times(N-p)
$$

The $\mathrm{X}$ values are F-distributed with 1 degree of freedom. In the given conditions all $\mathrm{X}$ $\leq 3.87(\mathrm{SSD} \leq 1.26 \cdot 10-5)$ are within the $95 \%$ confidence interval.

A set of IRRA spectra was subjected to principal component analysis (PCA) in order to identify subtle changes in the band shape correlated to hydration differences. The PCA was performed on vector-normalized spectra (to exclude contributions from intensity variations to the principal components) in the range of $1693-1770 \mathrm{~cm}^{-1}$. All spectra were pretreated as follows: i) a reference spectrum of a bare $\mathrm{D}_{2} \mathrm{O}$ subphase was subtracted from all spectra, ii) a $\mathrm{D}_{2} \mathrm{O}$ vapor spectrum was subtracted in a way to best reduce the contribution of vibrational-rotational bands in the spectral region of interest. iii) a polynomial rubber band baseline was subtracted from the spectra (Software OPUS, Bruker, Germany). All analyzed spectra were recorded in s-polarization, at 
various angles of incidence, $\varphi$. Four sets of spectra were analyzed in a common PCA: i) and ii) spectra of a DOPC:DOPE and of a DOPC:DOPE:PI(4,5) $\mathrm{P}_{2}$ monolayer before injection of protein $\left(20 \mathrm{mN} \mathrm{m}^{-1}\right.$, s-polarization, $\left.\varphi=40^{\circ}\right)$ and ii) and iv) spectra of the respective monolayers after injection of Cavin1 (1-190) $\left(c a .36 \mathrm{mN} \mathrm{m}^{-1}\right.$, s-polarization, $\left.\varphi=25-70^{\circ}\right)$. The PCA was performed using the princomp function of MATLAB (Math-Works Inc., Natick, MA, USA).

\section{Circular dichroism spectroscopy}

The secondary structure of Cavins was analyzed using a circular dichroism spectropolarimeter (JASCO J-810, Tokyo, Japan) at $25^{\circ} \mathrm{C}$ in the presence and absence of FOLCH liposomes. Liposome preparation were prepared as described for the cosedimentation assay. The final protein and liposome concentration was $3 \mu \mathrm{M}$ and 0.5 $\mathrm{mg} / \mathrm{ml}$, respectively, in $25 \mathrm{mM}$ HEPES, $150 \mathrm{mM} \mathrm{NaCl}(\mathrm{pH} 7.4)$. A cuvette with a 0.1 $\mathrm{cm}$ path length was used to acquire the spectra were measured from 190-260 nm by averaging 8 scans of each sample at a bandwidth of $2 \mathrm{~nm}$ and a scan rate of $50 \mathrm{~nm} / \mathrm{min}$. All samples were incubated for $5 \mathrm{~min}$ until equilibrium temperatures is reached. The buffer and liposome only spectra were measured as the background signals that were subtracted from protein signal.

\section{Computational simulations}

All simulations were performed using Gromacs 2018 software (44), and mouse Cavin1 HR1 domain structure (PDB: 4QKV) was used as the initial model (11). The membrane binding and insertion of HR1 domain, including trimer orientation and rotation, were predicated using coarse-grained molecular dynamics simulations (CG-MD). The hydrogen bonds and HR1 domain-membrane interactions were predicated by all-atom MD simulations. Details for software, scripts and parameters are described in the supplementary materials.

\section{Cell culture, transfection and live cell microscopy}

PC-3 cells (ECACC 90112714) were maintained in RPMI medium (GIBCO, Thermo

Fisher Scientific) supplemented with $10 \%$ foetal bovine serum and penicillin/streptomycin. For live cell, confocal imaging 400,000 cells were seeded on 1.5 high tolerance glass coverslips $25 \mathrm{~mm}$ (Warner Instruments, Hamden, CT, USA) $24 \mathrm{~h}$ prior to transfection. Caveolin1-RFP, Cavin1-GFP and Cavin1- $\triangle$ DR1-GFP were 
transiently transfected using Lipofectamine 2000 (Invitrogen) according to manufacturer's instructions 16 to $24 \mathrm{~h}$ before the experiment.

HeLa cells (ATCC-CRM-CCL-2) were grown in DMEM medium (GIBCO) supplemented with $10 \%$ foetal bovine serum (Invitrogen). For the generation of Flp-In TRex HeLa cell line, the C-terminal GFP tag was exchanged with the mCherry Cterminal tag in the CAV1-FlpIn T-REx HeLa cell line previously described (12). The cells were grown in DMEM (GIBCO) supplemented with $10 \%$ foetal bovine serum (Invitrogen), $100 \mu \mathrm{g} / \mathrm{ml}$ hygromycin B and $5 \mu \mathrm{g} / \mathrm{ml}$ blasticidin $\mathrm{S} \mathrm{HCl}$ (both GIBCO) for plasmid selection. For live cell, total internal reflection fluorescent (TIRF) imaging 80,000 cells were seeded on 1.5 high tolerance glass coverslips $25 \mathrm{~mm}$ (Warner Instruments) $24 \mathrm{~h}$ prior to transfection. Cavin1-GFP and Cavin1- $\triangle \mathrm{DR} 1-\mathrm{GFP}$ were transiently transfected as described above. CAV1-mCherry expression was induced with $0.5 \mathrm{ng} / \mathrm{ml}$ doxycycline hyclate (Sigma Aldrich) 12 to $18 \mathrm{~h}$ prior to the experiment. Cell lines were regularly tested for mycoplasma infection.

Live cell experiments were performed using a growth chamber $\left(37^{\circ} \mathrm{C}, 5 \% \mathrm{CO}_{2}\right)$ in connection to a Nikon Eclipse Ti-E inverted microscope microscope (Nikon Instruments Inc. Tokyo, Japan), equipped with DU897 ANDOR EMCCD camera (Oxford Instruments, Abingdon, UK) and a Nikon CFI Plan Apochromat 60x Oil (N.A 1.40) DIC objective and a Nikon CFI Plan Apochromat 100x (NA 1.49). The TIRF objective was controlled by NIS Elements interface (Nikon Instruments Inc.). Images were prepared using Image J (45) and Photoshop CS6 (Adobe Inc, San Jose, CA, USA). 


\section{Acknowledgments}

We acknowledge the Biochemical Imaging Center and Umeå Centre for Electron Microscopy at Umeå University and the National Microscopy Infrastructure, NMI for providing assistance in microscopy. MS analysis was performed at the Proteomics Core Facility of Sahlgrenska Academy, University of Gothenburg, Sweden. The computations/data handling were enabled by resources provided by the Swedish National Infrastructure for Computing (SNIC) at the Uppsala Multidisciplinary Center for Advanced Computational Science (UPPMAX), the Center for High Performance Computing (PDC), and the High-Performance Computing Center North (HPC2N) partially funded by the Swedish Research Council. This work was supported by the Kempe foundation, the Swedish Research Council, the Swedish Cancer Society Wallenberg Centre for Molecular Medicine and the Medical Faculty at Umeå University as well as the European Research Council.

\section{Funding:}

National Microscopy Infrastructure, NMI, VR-RFI 2019-00217 (EL, RL)

European Research Council grant 638965 (AK, SH, CASB)

Swedish Research Council through grant 2018-05973 (AK, SH, CASB)

Kempe Foundation, JCK-1657 (KCL, RL)

Swedish Research Council grant VR-NT 2017-04028 (RL)

\section{Author contributions:}

Conceptualization: KCL, EL, MH, RL

Methodological conceptualization: HP, CS, AS, SH, MH

Investigation: KCL, HP, EL, SH, AK, JM, CS, MH

Funding acquisition: $\mathrm{CSAB}, \mathrm{RL}$

Supervision: $\mathrm{MH}, \mathrm{RL}$

Writing — original draft: KCL, RL

Writing — review \& editing: KCL, HP, EL, CS, MH, RL 


\section{FIGURE LEGENDS}

Fig. 1. Cavin1 binding and insertion into model lipid membranes. (A) Scheme of the domain-structure of Cavin1 with DR and HR. White stripes mark undecad repeats. Post-translational modification sites are visualized in red, $\mathrm{P}$, phosphorylation; Ubiq, ubiquitination. The crystal structure of HR1 (4QKV) is displayed on top. (B) Liposome co-sedimentation of Cavin1. Cavin1 was incubated with or without FOLCH liposomes, centrifuged and supernatant $(\mathrm{S})$ and pellet $(\mathrm{P})$ fractions were analyzed by SDS-PAGE. Band intensities were quantified and data is shown as mean \pm SEM $(n=3)$. (C) Top; scheme of SLB formation. Bottom; QCM-D measurement showing shift in frequency $(\Delta \mathrm{F})$ (black line) and dissipation $(\Delta \mathrm{D})$ (red line) upon SLB formation. (D) Top; illustration of QCM-D set up. Bottom; QCM-D monitoring of Cavin1 adsorption to a SLB. The responses in $\Delta \mathrm{F}$ and $\Delta \mathrm{D}$ are corresponding to Cavin1 injection and buffer rinses as indicated. Grey dotted line shows extrapolation of protein desorption from first rinse (150 mM NaCl). (E) Top; scheme of protein adsorption experiments. Bottom; Cavin1 adsorption to DOPC:DOPE:PI(4,5) $\mathrm{P}_{2}$ monolayers. Cavin1 was injected underneath the film at $\pi_{0}=20 \mathrm{mN} \mathrm{m}^{-1}$ and $\Delta \pi$ recorded over time. (F) Cavin 1 adsorption to lipid monolayers was measured at different $\pi_{0}$. The MIP value was determined by extrapolation of the $\Delta \pi / \pi_{0}$ plot to the X-axis.

Fig. 2. The amount of Cavin1 (1-190) inserted into membranes is $\mathrm{PI}(4,5) \mathrm{P}_{2}$ dependent. (A) Liposome co-sedimentation assay. Cavin1 (1-190) or Cavin1 (191392) were incubated with or without FOLCH liposomes, centrifuged and supernatant (S) and pellet (P) fractions were analyzed by SDS-PAGE. Band intensities were quantified and data is shown as mean \pm SEM (n=3). (B) Cavin1 (1-190) adsorption to DOPC:DOPE:PI(4,5)P2 monolayers. Cavin1 (1-190) was injected at $\pi_{0}=20 \mathrm{mN} \mathrm{m}^{-1}$ and $\Delta \pi$ recorded over time. (C) Cavin1 (1-190) adsorption to lipid monolayers was measured at different $\pi_{0}$. MIP was determined by extrapolation of $\Delta \pi / \pi_{0}$ plot to x-axis. (D) IRRA spectra (1790-1590 $\left.\mathrm{cm}^{-1}\right)$ of DOPC:DOPE:PI(4,5)P 2 at $\pi_{0}=20 \mathrm{mN} \mathrm{m}^{-1}$. The $\mathrm{C}=\mathrm{O}$ vibrational band $\left(\sim 1730 \mathrm{~cm}^{-1}\right)$ originates from lipid ester groups. The amide I' band $\left(\sim 1640 \mathrm{~cm}^{-1}\right)$ indicates Cavin1 (1-190) adsorption after injection into the subphase. Spectra were acquired with p-polarized light at an angle of incidence of $40^{\circ}$. (E) $\mathrm{PCA}$ in the $\mathrm{C}=\mathrm{O}$ vibrational region based on IRRA spectra of 
DOPC:DOPE:PI(4,5)P2 and DOPC:DOPE monolayers before and after adsorption of Cavin1 (1-190). PC1 represents the extent of carbonyl group hydration (76\% of total variance), while PC2 (10\%) showed no additional systematic changes. (F) Amount of protein adsorbed to the monolayer. Ratios of integral intensity of amide I' and $\mathrm{C}=\mathrm{O}$ bands are shown as a function of the angles of incidence for indicated lipid monolayers. Dotted lines display the mean of each data set.

Fig. 3. Detail identification of membrane insertion of Cavin1 N-terminus. (A) Top; Scheme of the Cavin1 N-terminal domains with defined amino acids numbers: DR1 (144), HR1 (45-156) and DR2 (156-210), in comparison to the N-terminal truncated mutants. Bottom; Far-UV CD spectra (200-260 nm) of Cavin1 truncated proteins as indicated. (B) Adsorption of Cavin1 truncates to DOPC:DOPE:PI(4,5) $\mathrm{P}_{2}$ monolayers. Proteins were injected at $\pi_{0}=20 \mathrm{mN} \mathrm{m}^{-1}$ and $\Delta \pi$ recorded over time. (C) Quantification of $\Delta \pi$ recorded in (B) at $\mathrm{t}=20 \mathrm{~min}$. Data is shown as mean $\pm \mathrm{SEM}$. (D) Conformational change of Cavin1 N-terminus upon membrane interaction. Far-UV CD spectra (195$255 \mathrm{~nm})$ of Cavin1 (1-155) and (1-190) with or without liposomes. The ratio between 222 and $208 \mathrm{~nm}$ ellipticities $\left(\left[\theta_{222}\right] /\left[\theta_{208}\right]\right)$ is plotted for estimation of $\alpha$-helical content.

(E) Frequency shift $(\Delta \mathrm{F})$ and $(\mathbf{F})$ Dissipation shift $(\Delta \mathrm{D})$ as a result of adsorption of Cavin1 (1-190) and (44-190) onto SLBs measured by QCM-D. After a stable SLB formed, proteins were injected into the system and adsorbed upon SLB (grey area), and rinsed with buffer. (G) Relative softness plot $(\Delta D /-\Delta F$ vs $-\Delta F)$ showing changes in surface softness during protein adsorption and desorption. Arrows indicate the curves direction over time.

Fig. 4. Cavin1 (1-190) adsorbs to membranes with an inclination of $20-25^{\circ}$ as determined by IRRAS. (A) Experimental and best fitting simulated IRRA spectra of Cavin1 (1-190) adsorbed to a DOPC/DOPE/PI(4,5) $\mathrm{P}_{2}$ monolayer, at various angles of incidence $\varphi$ and polarizations. Vertical dotted lines indicate the band components used for simulation. The best fit was achieved with a helix inclination angle of $\gamma=22.5 \pm$ $2.5^{\circ}$ with respect to the lipid interface. (B) The quality of the fit was assessed as sum square deviation between experimental and simulated spectra at various $\gamma$. The minimum is indicated by a red $\mathrm{x}$-axis tick. The $95 \%$ confidence interval of the minimum is shown in blue. Red vertical lines mark a slightly larger range of plausible HR1 
orientations considering uncertainties in experiment, model assumptions and data treatment. The upper $\mathrm{x}$-axis shows the inclination angles of the complete HR1 trimer that correspond to the individual helices' inclination angles. Note that their relation is not linear. (C) Scheme of HR1 trimer orientation at lipid monolayer. The most probable orientation is shown in dark gray and outlined by blue dotted lines. Red dotted lines indicate the range of inclination angles that plausibly explain the measured spectra.

Fig. 5. The HR1 domain initially binds via any of the three helices and orients parallel to the membrane interphase. (A) CG-MD simulations of HR1 membrane binding. Helices A, B and C are color-coded as blue, red and gray, respectively. Membranes consisted of DOPC (silver), DOPE (green) and PI(4,5)P 2 (purple), where head group beads are shown in blue and orange. Upper panel; Probabilities for initial binding between $\mathrm{N}$ - or $\mathrm{C}$-terminal lysines and $\mathrm{PI}(4,5) \mathrm{P}_{2}$ are given. Lower panel; HR1 was horizontally bound at the end of all simulations $(2 \mu \mathrm{s})$. (B) Representative bottomup view from the membrane for all-atom simulations of HR1. The membrane composition was identical to (A) but only $\mathrm{PI}(4,5) \mathrm{P}_{2}$ (cyan) is shown for clarity. Residues highlighted in yellow were solvent-inaccessible and engaged in hydrogen bonding with the membrane, predominantly with $\mathrm{PI}(4,5) \mathrm{P}_{2}$. All-atom simulations of (C) HR1 rotation around its helical axis and (D) associated free energy $(\Delta \mathrm{G})$ of proteinmembrane interactions at different rotation angles. Lower panel in $(C)$ shows the axial views at different angles, where maxima and minima of $\Delta \mathrm{G}$ are color-coded in green and red, respectively, and correspond to maxima and minima energy states seen in (D). (E) Electrostatic surface potential of HR1 (red, negative charge; blue, positive charge). (F) Representative snapshot of a monomeric HR1 domain (red) inserted into the membrane containing $\mathrm{PI}(4,5) \mathrm{P}_{2}$ (purple).

Fig. 6. N-terminal DR1 domain facilitates membrane insertion of Cavin1. (A) Immunoblot of caveolar proteins from caveolae enriched membrane (CEM) fractions following mock treatment (control) or treatment with $4 \mathrm{M} \mathrm{NaCl}$ or $10 \mathrm{mM}$ Methyl- $\beta$ cyclodextrin (M $\beta C D$ ) or $60 \mathrm{mM}$ octyl glucoside. CEM fractions was analyzed by using antibodies against EHD2, Cavin1, Cavin2, Cavin3 and CAV1 (B) Representative confocal microscopy images of PC-3 cells transfected with fluorescently tagged CAV1 (CAV1-RFP, red), full-length Cavin1 (Cavin1-GFP, green) or Cavin1- $\triangle$ DR1 (Cavin1- 
$\Delta$ DR1-GFP, green). Focal plane in the top panel shows protein localization at the basal membrane and in the bottom panel protein localization in the mid-section of a cell. White arrows highlight intracellular CAV1 structures devoid of Cavin1- $\triangle \mathrm{DR} 1-\mathrm{GFP}$. Scale bar $=10 \mu \mathrm{m}$ 


\section{REFERENCES}

1. R. G. Parton, M. M. Kozlov, N. Ariotti, Caveolae and lipid sorting: Shaping the cellular response to stress. J. Cell Biol. 219, (2020).

2. R. G. Parton, K.-A. McMahon, Y. Wu, Caveolae: Formation, dynamics, and function. Curr. Opin. Cell Biol. 65, 8-16 (2020).

3. R. G. Parton, M. A. Del Pozo, S. Vassilopoulos, I. R. Nabi, S. Le Lay, R. Lundmark, A. K. Kenworthy, A. Camus, C. M. Blouin, W. C. Sessa, C. Lamaze, Caveolae: The FAQs. Traffic 21, 181-185 (2020).

4. M. Hubert, E. Larsson, N. V. G. Vegesna, M. Ahnlund, A. I. Johansson, L. W. K. Moodie, R. Lundmark, Lipid accumulation controls the balance between surface connection and scission of caveolae. Elife 9, e55038 (2020).

5. Y. Zhou, N. Ariotti, J. Rae, H. Liang, V. Tillu, S. Tee, M. Bastiani, A. T. Bademosi, B. M. Collins, F. A. Meunier, J. F. Hancock, R. G. Parton, Caveolin1 and cavin1 act synergistically to generate a unique lipid environment in caveolae. J. Cell Biol. 220, (2021).

6. A. Ludwig, G. Howard, C. Mendoza-Topaz, T. Deerinck, M. Mackey, S. Sandin, M. H. Ellisman, B. J. Nichols, Molecular composition and ultrastructure of the caveolar coat complex. PLoS Biol. 11, e1001640 (2013).

7. Y. Gambin, N. Ariotti, K. A. McMahon, M. Bastiani, E. Sierecki, O. Kovtun, M. E. Polinkovsky, A. Magenau, W. Jung, S. Okano, Y. Zhou, N. Leneva, S. Mureev, W. Johnston, K. Gaus, J. F. Hancock, B. M. Collins, K. Alexandrov, R. G. Parton, Single-molecule analysis reveals self assembly and nanoscale segregation of two distinct cavin subcomplexes on caveolae. Elife 3, e01434 (2014).

8. K. G. Rothberg, J. E. Heuser, W. C. Donzell, Y. S. Ying, J. R. Glenney, R. G. Anderson, Caveolin, a protein component of caveolae membrane coats. Cell $\mathbf{6 8}$, 673-682 (1992).

9. B. Han, J. C. Porta, J. L. Hanks, Y. Peskova, E. Binshtein, K. Dryden, D. P. Claxton, H. S. McHaourab, E. Karakas, M. D. Ohi, A. K. Kenworthy, Structure and assembly of CAV1 $8 \mathrm{~S}$ complexes revealed by single particle electron microscopy. Sci. Adv. 6, eabc6185 (2020).

10. A. R. Busija, H. H. Patel, P. A. Insel, Caveolins and cavins in the trafficking, maturation, and degradation of caveolae: implications for cell physiology. Am. J. Physiol. Cell Physiol. 312, C459-C477 (2017).

11. O. Kovtun, V. A. Tillu, W. Jung, N. Leneva, N. Ariotti, N. Chaudhary, R. A. Mandyam, C. Ferguson, G. P. Morgan, W. A. Johnston, S. J. Harrop, K. Alexandrov, R. G. Parton, B. M. Collins, Structural insights into the organization of the cavin membrane coat complex. Dev. Cell 31, 405-419 (2014). 
12. J. Mohan, B. Moren, E. Larsson, M. R. Holst, R. Lundmark, Cavin3 interacts with cavin 1 and caveolin1 to increase surface dynamics of caveolae. J. Cell Sci. 128, 979-991 (2015).

13. M. Stoeber, P. Schellenberger, C. A. Siebert, C. Leyrat, A. Helenius, K. Grunewald, Model for the architecture of caveolae based on a flexible, net-like assembly of Cavin1 and Caveolin discs. Proc. Natl. Acad. Sci. U.S.A. 113, E8069-E8078 (2016).

14. A. Ludwig, B. J. Nichols, S. Sandin, Architecture of the caveolar coat complex. J. Cell Sci. 129, 3077-3083 (2016).

15. M. Hubert, E. Larsson, R. Lundmark, Keeping in touch with the membrane; protein- and lipid-mediated confinement of caveolae to the cell surface. Biochem. Soc. Trans. 48, 155-163 (2020).

16. A. Fujita, J. Cheng, K. Tauchi-Sato, T. Takenawa, T. Fujimoto, A distinct pool of phosphatidylinositol 4,5-bisphosphate in caveolae revealed by a nanoscale labeling technique. Proc. Natl. Acad. Sci. U.S.A. 106, 9256-9261 (2009).

17. V. A. Tillu, Y.-W. Lim, O. Kovtun, S. Mureev, C. Ferguson, M. Bastiani, K.A. McMahon, H. P. Lo, T. E. Hall, K. Alexandrov, B. M. Collins, R. G. Parton, A variable undecad repeat domain in cavin1 regulates caveola formation and stability. EMBO Rep. 19, e45775 (2018).

18. V. A. Tillu, J. Rae, Y. Gao, N. Ariotti, M. Floetenmeyer, O. Kovtun, K.-A. McMahon, N. Chaudhary, R. G. Parton, B. M. Collins, Cavin1 intrinsically disordered domains are essential for fuzzy electrostatic interactions and caveola formation. Nat. Commun. 12, 931 (2021).

19. V. A. Tillu, O. Kovtun, K.-A. McMahon, B. M. Collins, R. G. Parton, A phosphoinositide-binding cluster in cavin 1 acts as a molecular sensor for cavin 1 degradation. Mol. Biol. Cell 26, 3561-3569 (2015).

20. C. A. Keller, B. Kasemo, Surface specific kinetics of lipid vesicle adsorption measured with a quartz crystal microbalance. Biophys. J. 75, 1397-1402 (1998).

21. R. Richter, A. Mukhopadhyay, A. Brisson, Pathways of lipid vesicle deposition on solid surfaces: a combined QCM-D and AFM study. Biophys. J. 85, 30353047 (2003).

22. N.-J. Cho, C. W. Frank, B. Kasemo, F. Höök, Quartz crystal microbalance with dissipation monitoring of supported lipid bilayers on various substrates. Nat. Protoc. 5, 1096-1106 (2010).

23. A. Blume, A. Kerth, Peptide and protein binding to lipid monolayers studied by FT-IRRA spectroscopy. Biochim. Biophys. Acta 1828, 2294-2305 (2013).

24. É. Boisselier, P. Calvez, É. Demers, L. Cantin, C. Salesse, Influence of the Physical State of Phospholipid Monolayers on Protein Binding. Langmuir 28, 9680-9688 (2012). 
25. P. Calvez, S. Bussières, D. Eric, C. Salesse, Parameters modulating the maximum insertion pressure of proteins and peptides in lipid monolayers. Biochimie 91, 718-733 (2009).

26. A. Blume, A comparative study of the phase transitions of phospholipid bilayers and monolayers. Biochim. Biophys. Acta 557, 32-44 (1979).

27. A. Blume, W. Huebner, G. Messner, Fourier transform infrared spectroscopy of 13C:O labeled phospholipids hydrogen bonding to carbonyl groups. Biochemistry 27, 8239-8249 (1988).

28. M. Rabe, C. Schwieger, H. R. Zope, F. Versluis, A. Kros, Membrane Interactions of Fusogenic Coiled-Coil Peptides: Implications for Lipopeptide Mediated Vesicle Fusion. Langmuir 30, 7724-7735 (2014).

29. W. Ding, M. Palaiokostas, W. Wang, M. Orsi, Effects of Lipid Composition on Bilayer Membranes Quantified by All-Atom Molecular Dynamics. J. Phys. Chem. B 119, 15263-15274 (2015).

30. W. J. Chang, Y. S. Ying, K. G. Rothberg, N. M. Hooper, A. J. Turner, H. A. Gambliel, J. De Gunzburg, S. M. Mumby, A. G. Gilman, R. G. Anderson, Purification and characterization of smooth muscle cell caveolae. J. Cell Biol. 126, 127-138 (1994).

31. F. Meng, B. Joshi, I. R. Nabi, Galectin-3 Overrides PTRF/Cavin-1 Reduction of PC3 Prostate Cancer Cell Migration. PLoS One 10, e0126056 (2015).

32. R. G. Parton, V. Tillu, K.-A. McMahon, B. M. Collins, Key phases in the formation of caveolae. Curr. Opin. Cell Biol. 71, 7-14 (2021).

33. M. Giménez-Andrés, A. Čopič, B. Antonny, The Many Faces of Amphipathic Helices. Biomolecules 8, 45 (2018).

34. S. Vanni, L. Vamparys, R. Gautier, G. Drin, C. Etchebest, P. F. J. Fuchs, B. Antonny, Amphipathic lipid packing sensor motifs: probing bilayer defects with hydrophobic residues. Biophys. J. 104, 575-584 (2013).

35. A. Auerswald, T. Gruber, J. Balbach, A. Meister, Lipid-Dependent Interaction of Human N-BAR Domain Proteins with Sarcolemma Mono- and Bilayers. Langmuir 36, 8695-8704 (2020).

36. C. Schwieger, A. Meister, S. Daum, A. Blume, K. Bacia, Binding of the GTPase Sar1 to a Lipid Membrane Monolayer: Insertion and Orientation Studied by Infrared Reflection-Absorption Spectroscopy. Polymers 9, 612 (2017).

37. D. Westphal, R. M. Kluck, G. Dewson, Building blocks of the apoptotic pore: how Bax and Bak are activated and oligomerize during apoptosis. Cell. Death. Differ. 21, 196-205 (2014).

38. A. H. Lystad, S. R. Carlsson, L. R. de la Ballina, K. J. Kauffman, S. Nag, T. Yoshimori, T. J. Melia, A. Simonsen, Distinct functions of ATG16L1 isoforms in membrane binding and LC3B lipidation in autophagy-related processes. Nat. Cell Biol. 21, 372-383 (2019). 
39. E. Larsson, M. Hubert, R. Lundmark, "Analysis of Protein and Lipid Interactions Using Liposome Co-sedimentation Assays" in Caveolae: Methods and Protocols, C. M. Blouin, Ed. (Springer US, New York, NY, 2020), pp. 119127.

40. V. L. Kuzmin, V. P. Romanov, A. V. Mikhailov, Light-Reflection on the Boundary of Liquid-Systems and Surface-Layer Structure. Opt. Spektrosk. 73, 3-47 (1992).

41. C. Schwieger, B. Chen, C. Tschierske, J. Kressler, A. Blume, Organization of T-Shaped Facial Amphiphiles at the Air/Water Interface Studied by Infrared Reflection Absorption Spectroscopy. J. Phys. Chem. B 116, 12245-12256 (2012).

42. J. E. Bertie, M. K. Ahmed, H. H. Eysel, Infrared intensities of liquids. 5. Optical and dielectric constants, integrated intensities, and dipole moment derivatives of water and water-d2 at 22.degree.C. J. Phys. Chem. 93, 2210-2218 (1989).

43. T. Buffeteau, E. Le Calvez, S. Castano, B. Desbat, D. Blaudez, J. Dufourcq, Anisotropic Optical Constants of $\alpha$-Helix and $\beta$-Sheet Secondary Structures in the Infrared. J. Phys. Chem. B 104, 4537-4544 (2000).

44. M. J. Abraham, T. Murtola, R. Schulz, S. Páll, J. C. Smith, B. Hess, E. Lindahl, GROMACS: High performance molecular simulations through multi-level parallelism from laptops to supercomputers. SoftwareX 1-2, 19-25 (2015).

45. D. Darriba, G. L. Taboada, R. Doallo, D. Posada, jModelTest 2: more models, new heuristics and parallel computing. Nat. Methods 9, 772-772 (2012). 


\section{Figure 1}

A

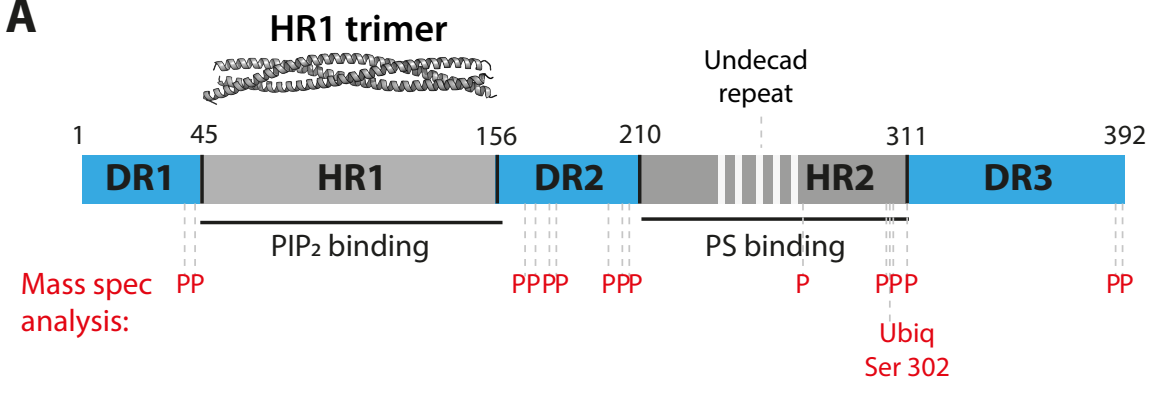

B - $\frac{\text { Liposomes }}{\mathrm{S} P}+\frac{\text { Liposomes }}{\mathrm{S} P}$

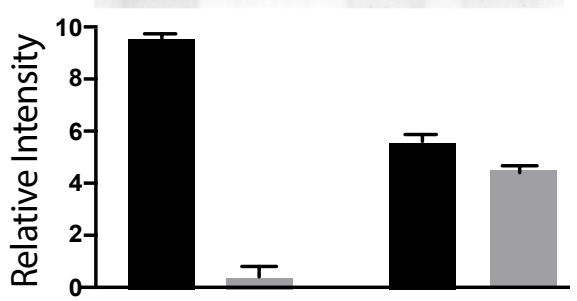

D

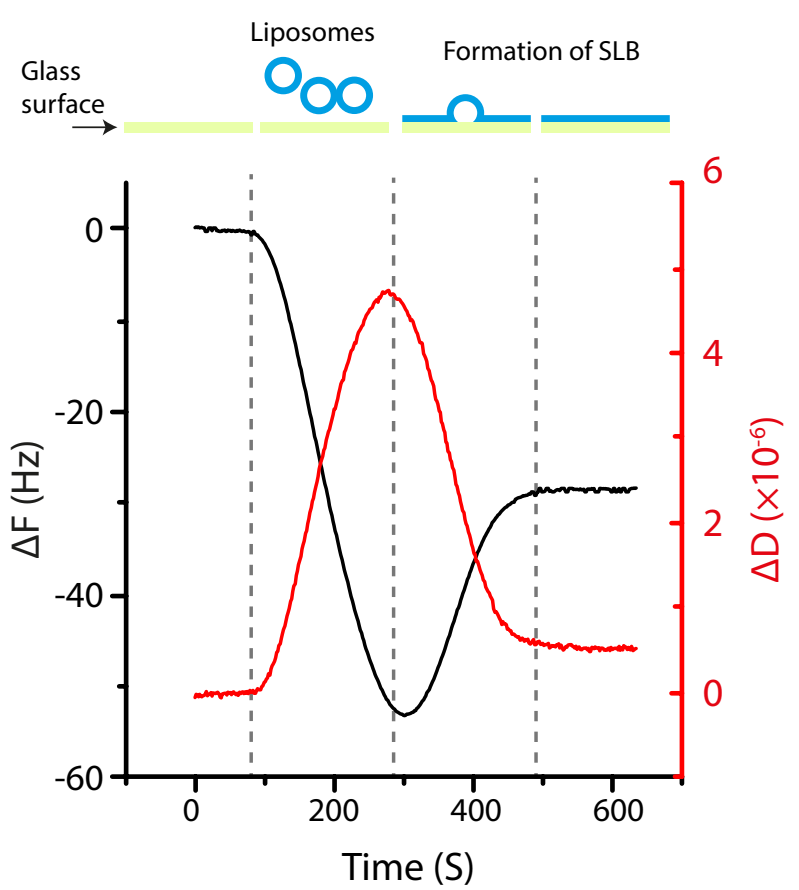

E

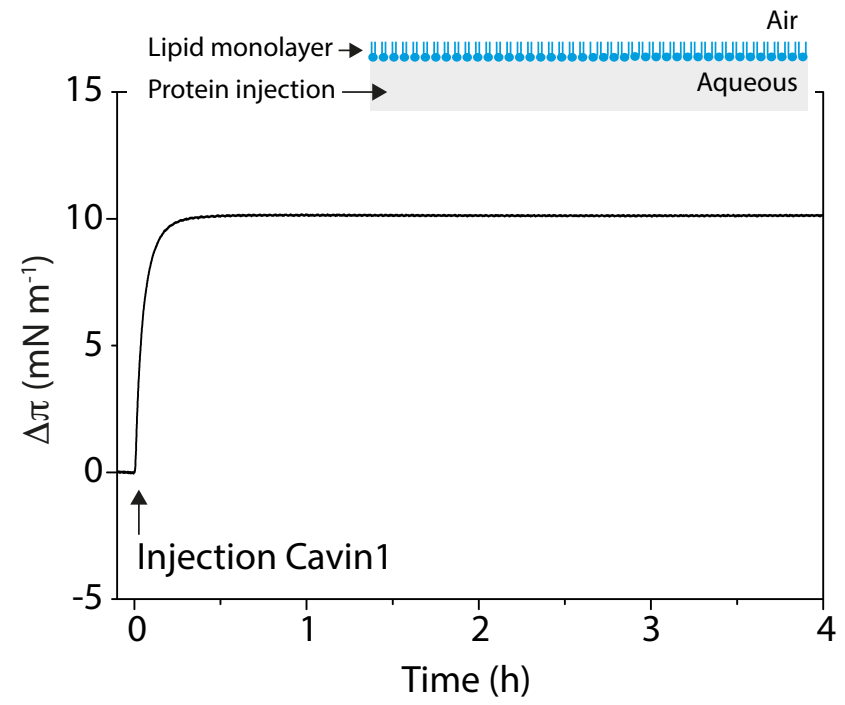

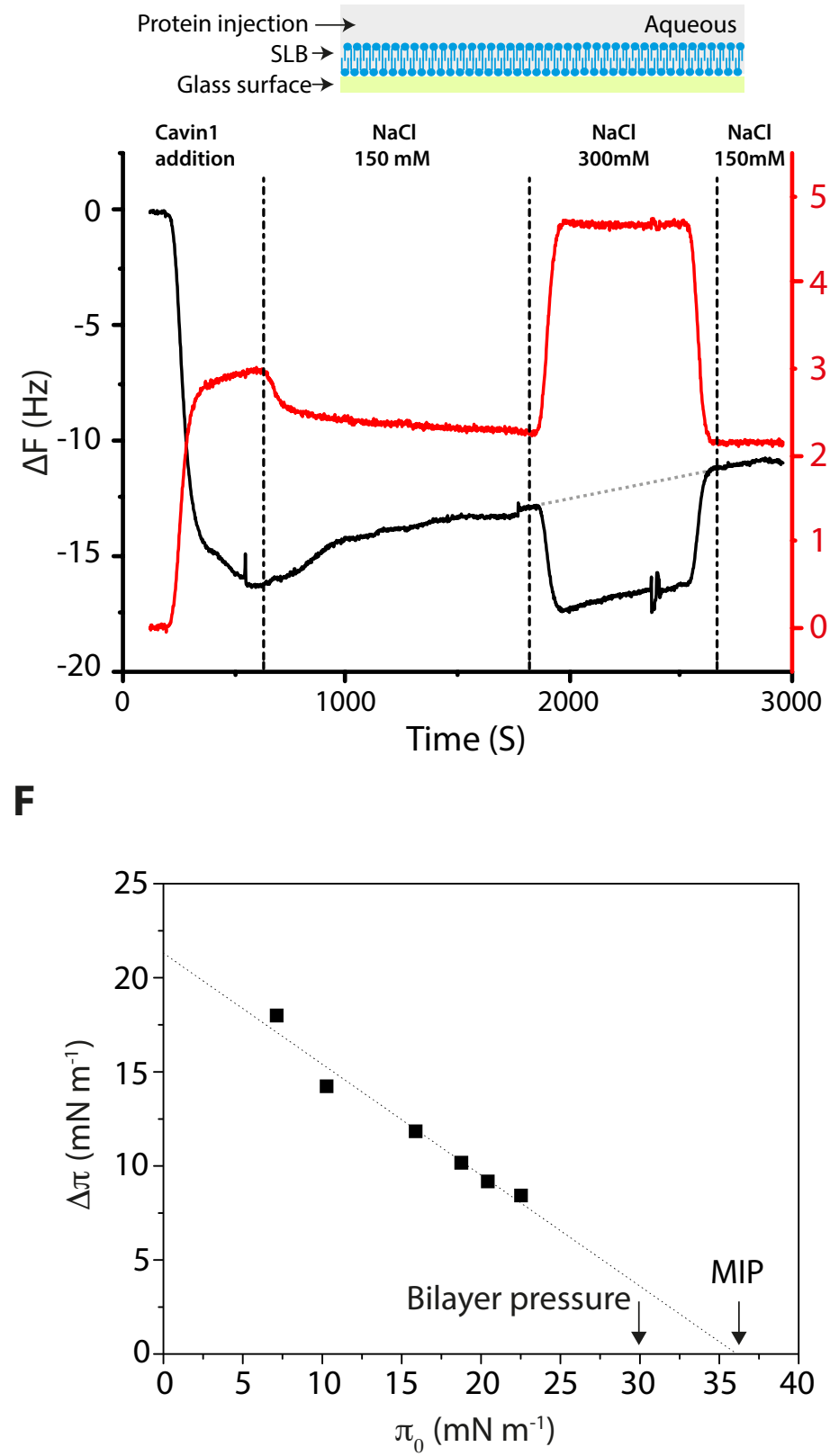


Figure 2

A

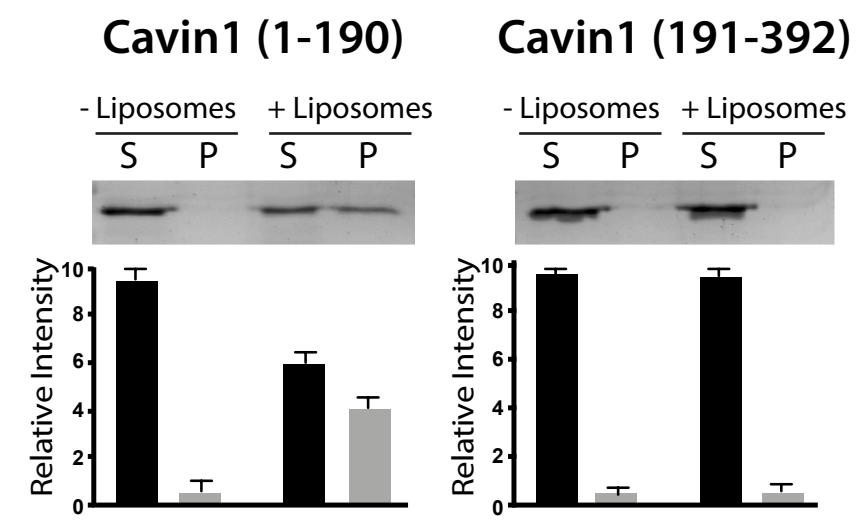

C

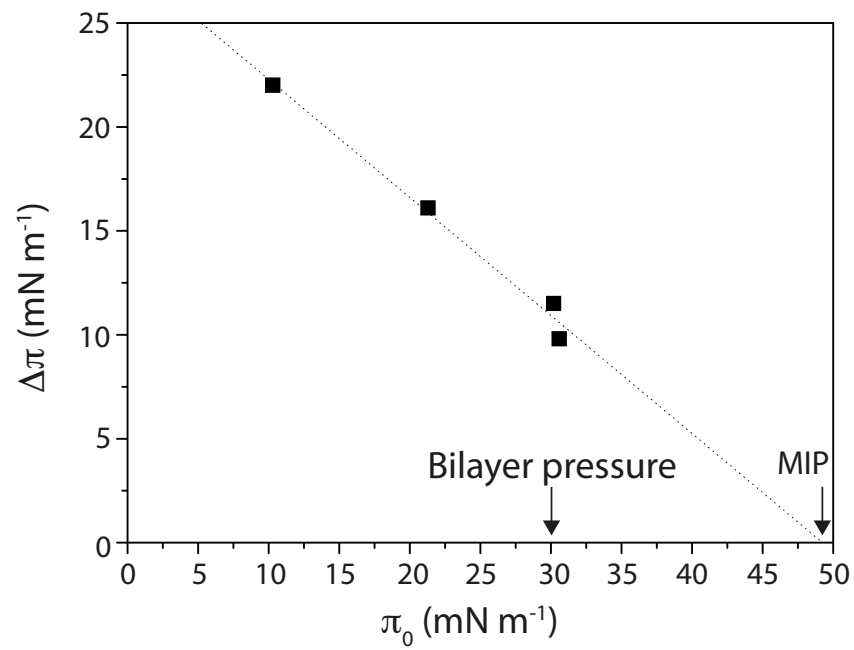

E

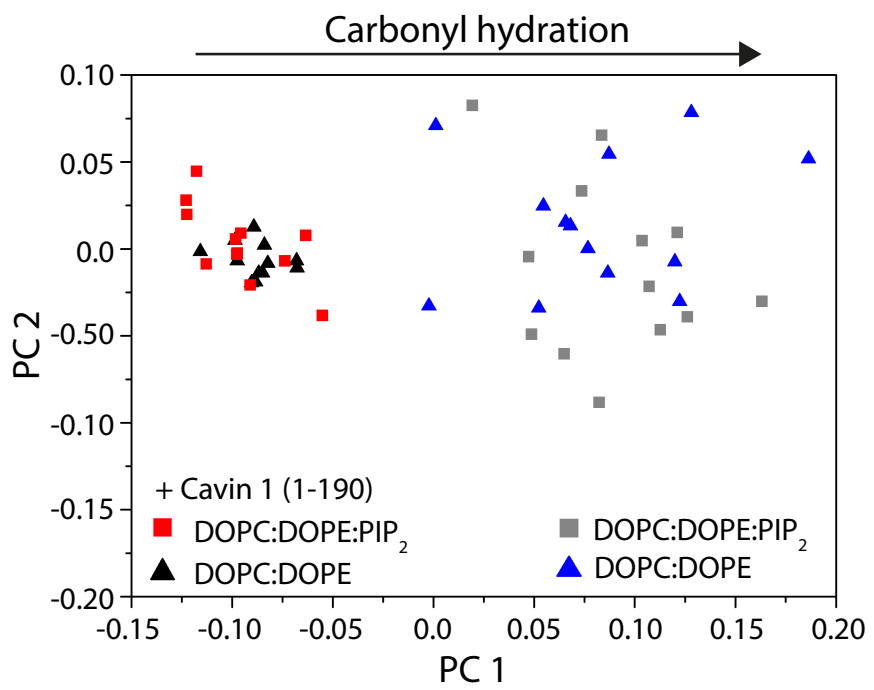

B

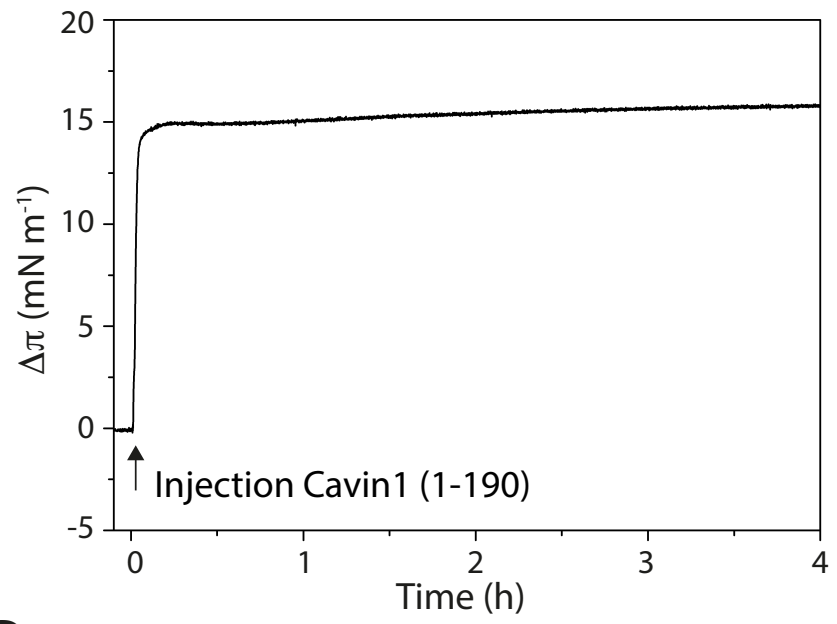

D

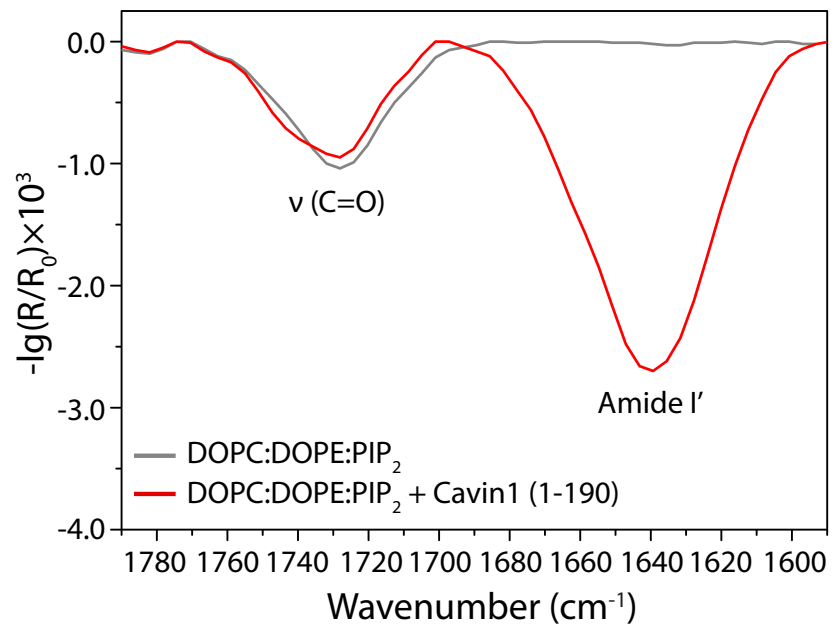

F

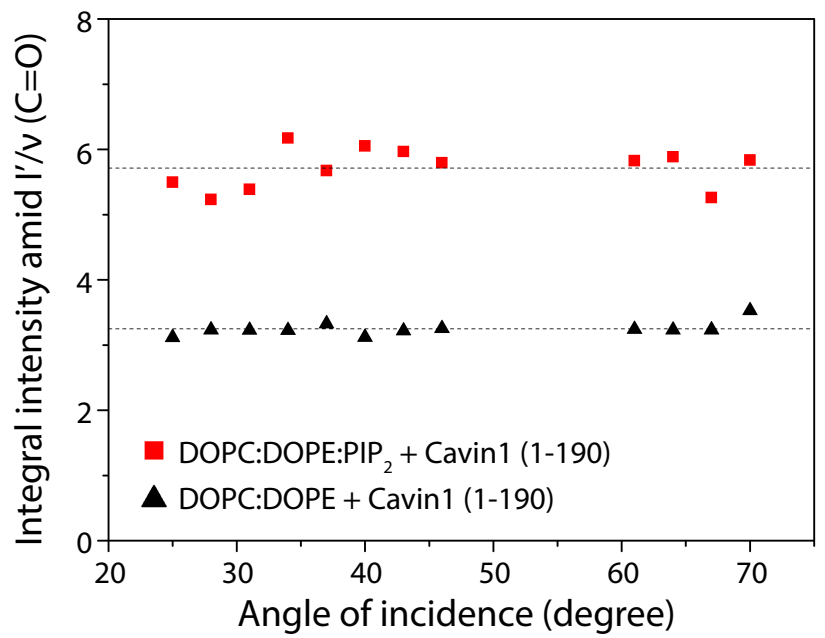


bioBxiv preprint doi: https://doi.org/10.1101/2021.03.23.436578; this version posted March 25, 2021. The copyright holder for this preprint (which was not certified by peer review) is the author/funder. All rights reserved. No reuse allowed without permission.

A

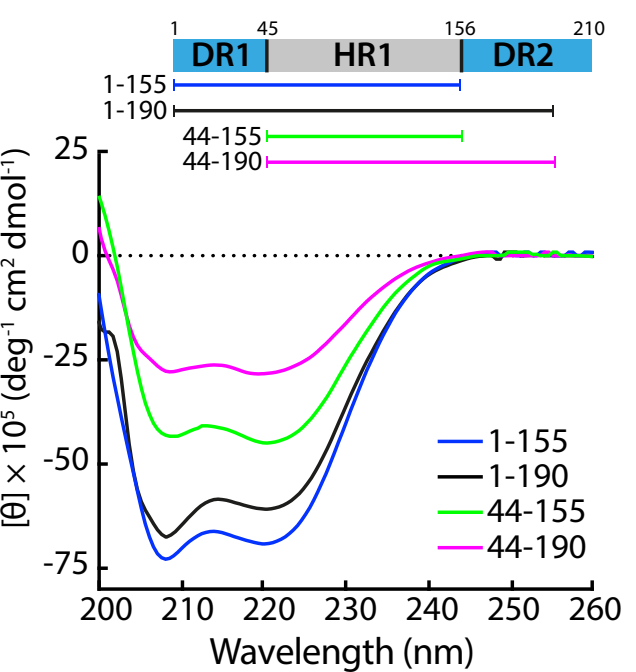

D

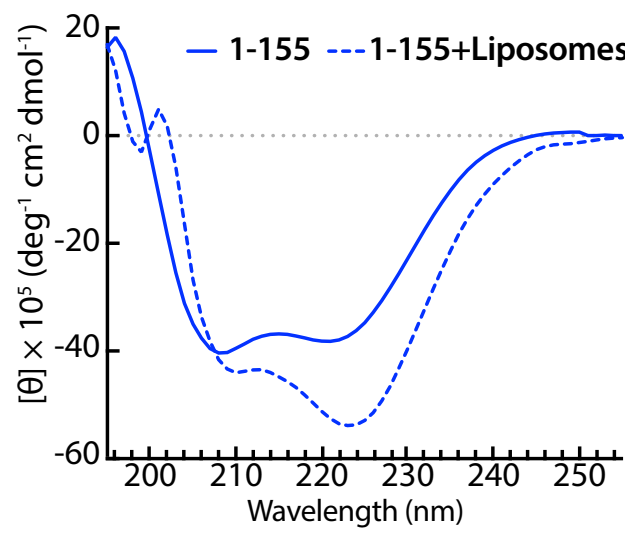

E

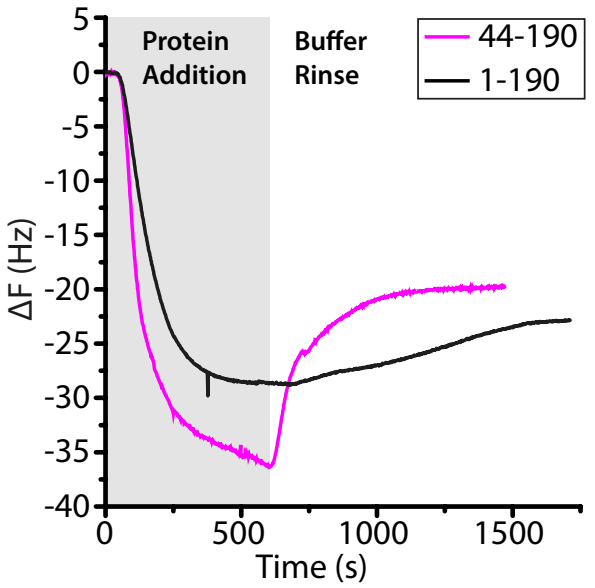

B

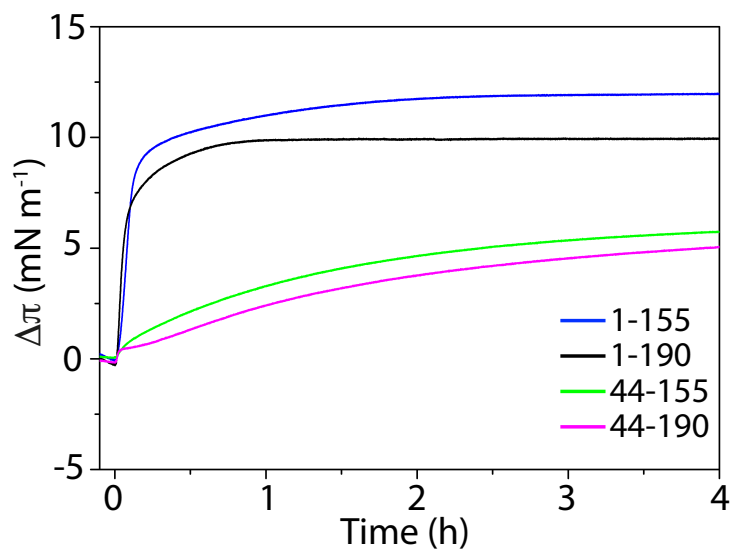

C

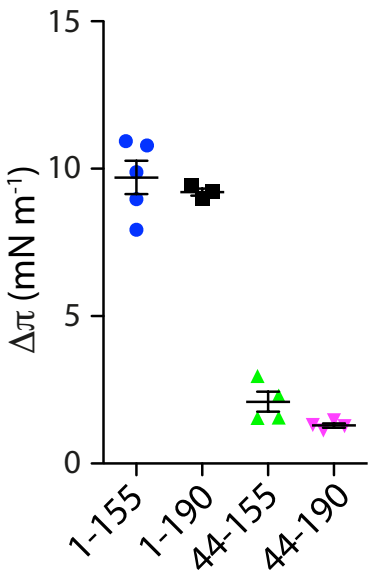

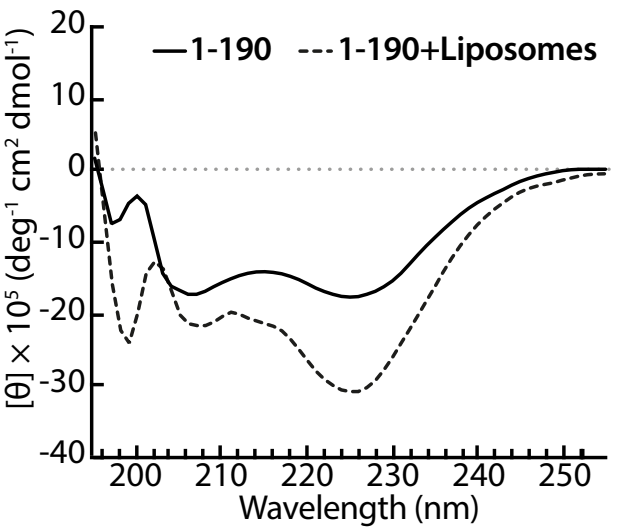

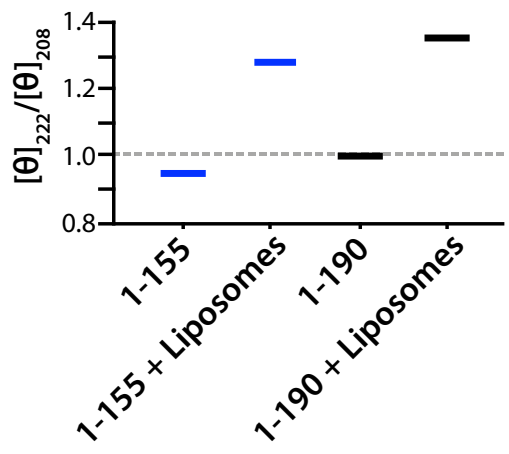

F

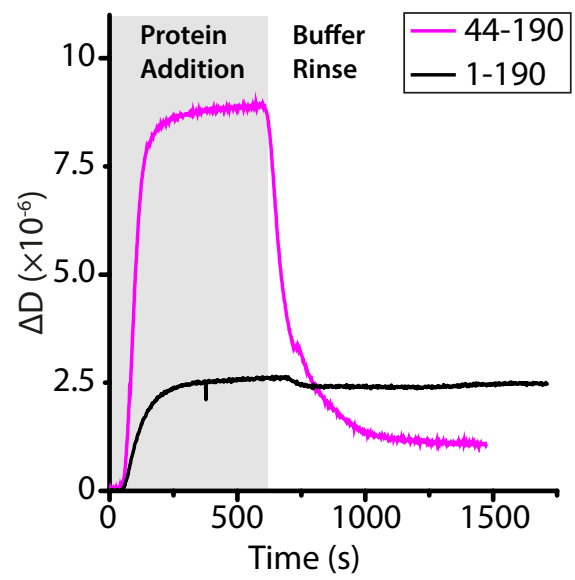

G

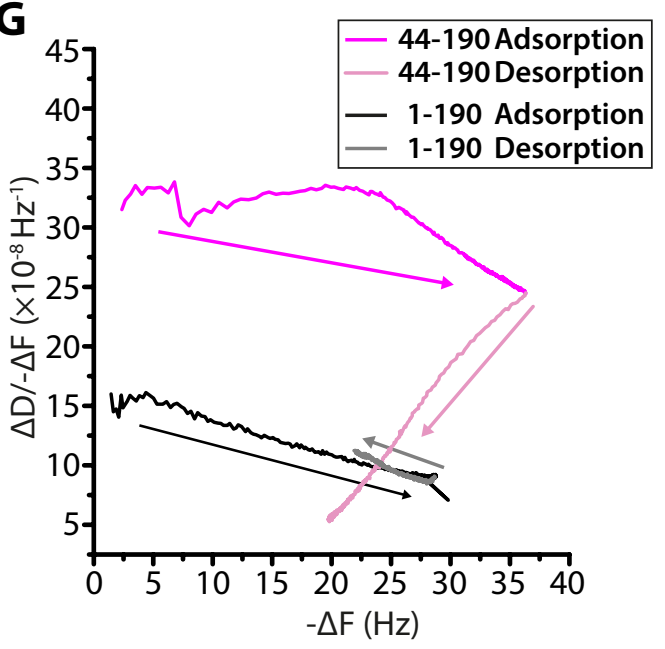




\section{Figure 4}

A

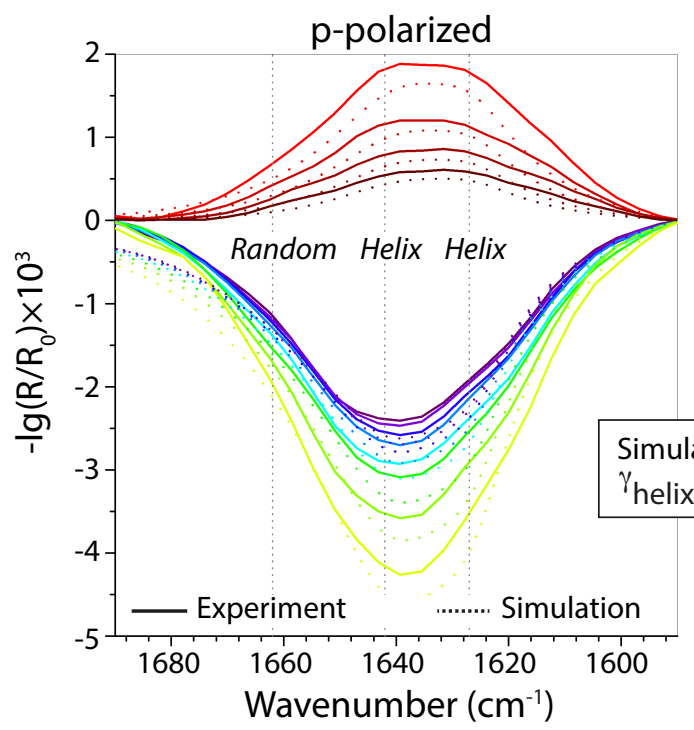

B

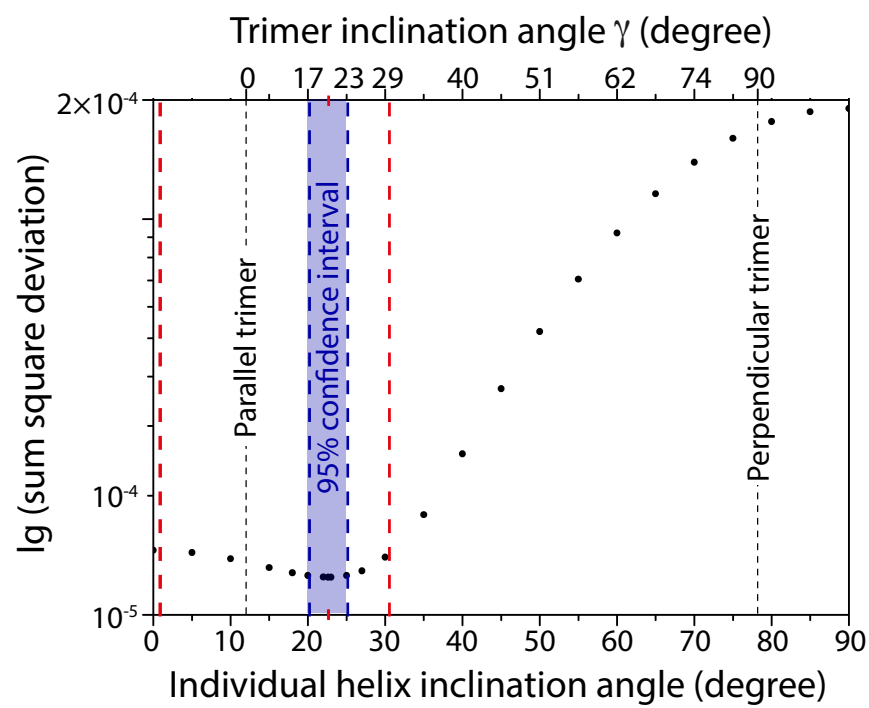

s-polarized

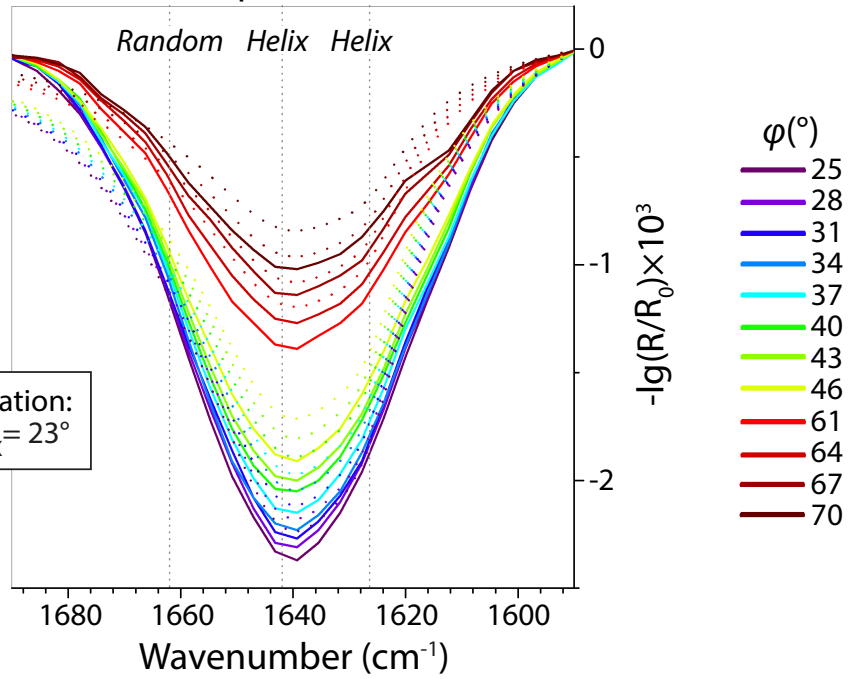

C

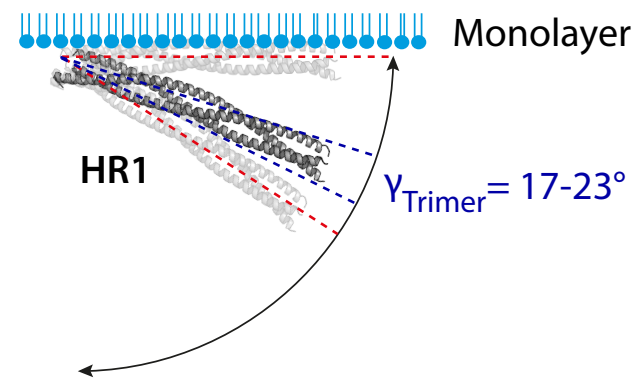



Fig ure $\mathbf{5}^{\text {(which was not certified by peer review) is the author/funder. All rights reserved. No reuse allowed without permission. }}$

A

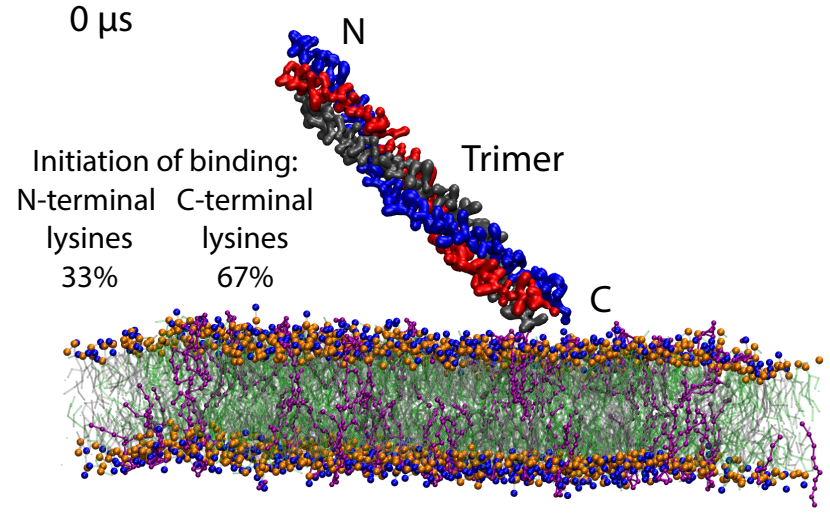

$2 \mu \mathrm{s}$

Trimer

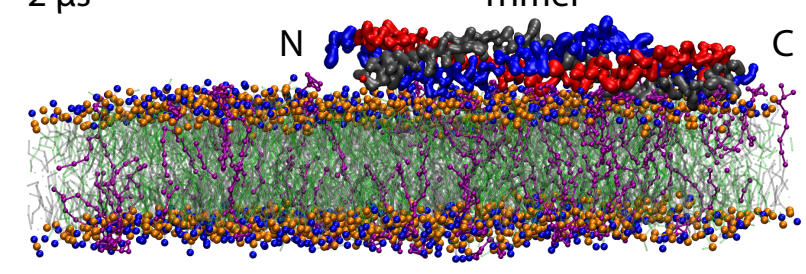

C
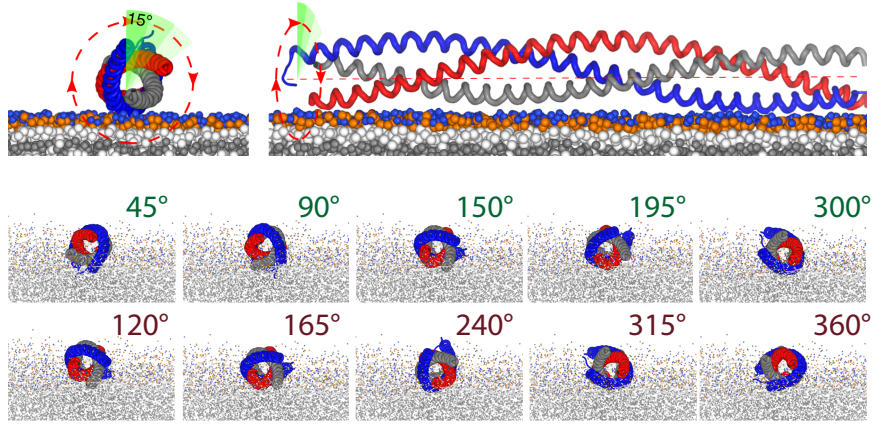

E
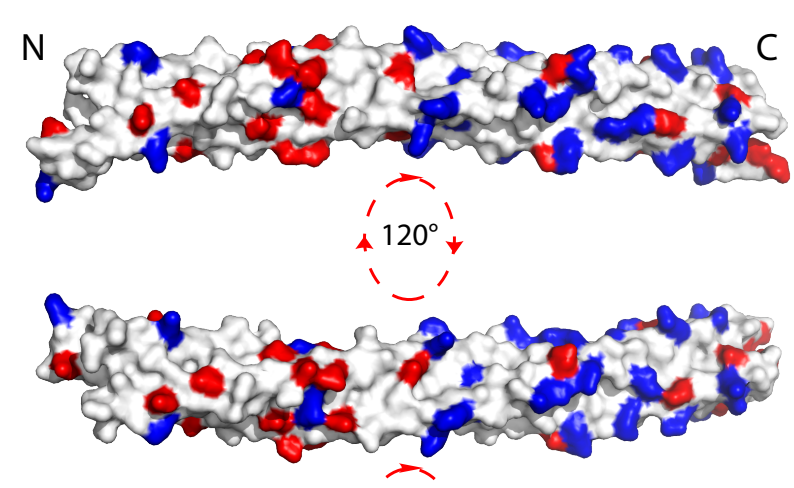

4 $120^{\circ}$

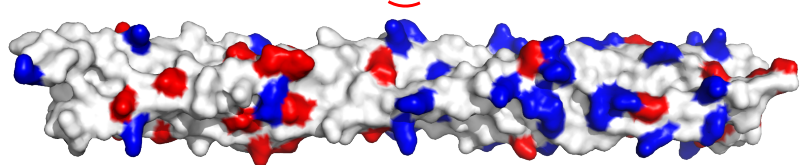

B

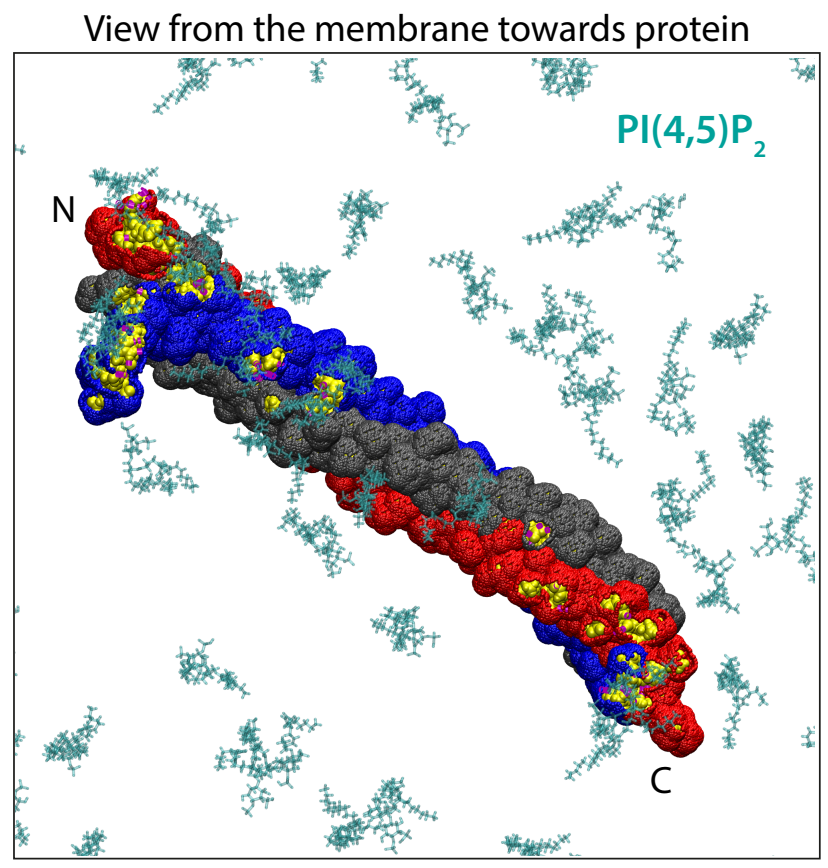

D

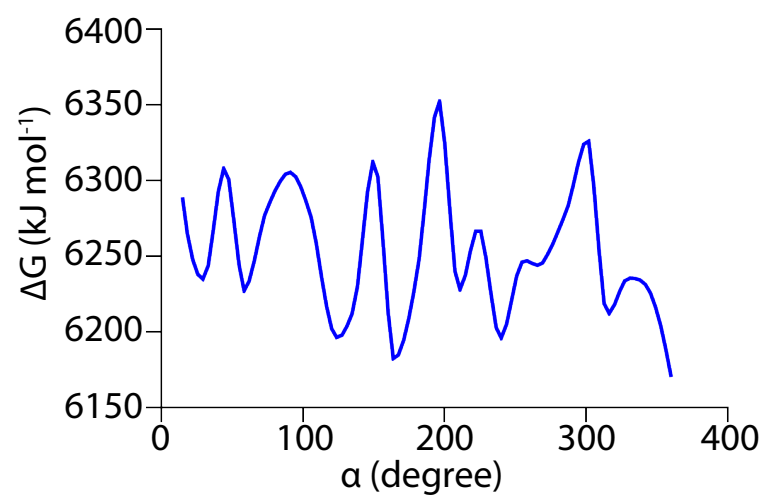

$\mathbf{F}$

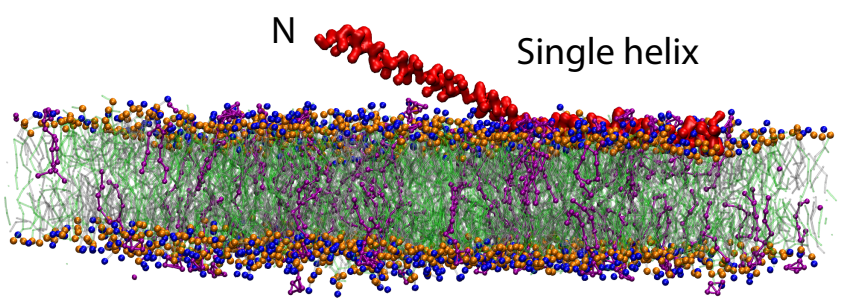




\section{Figure 6}

A

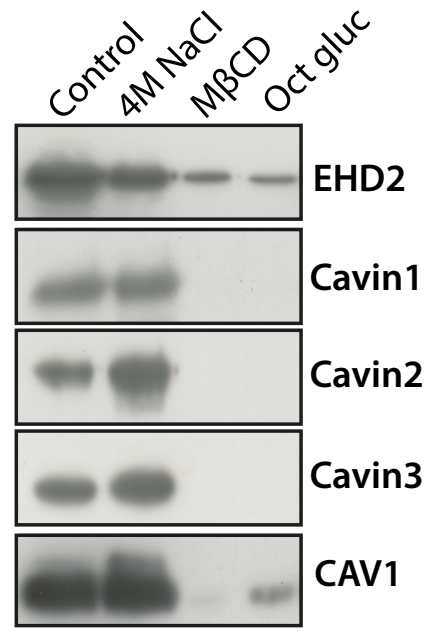

B
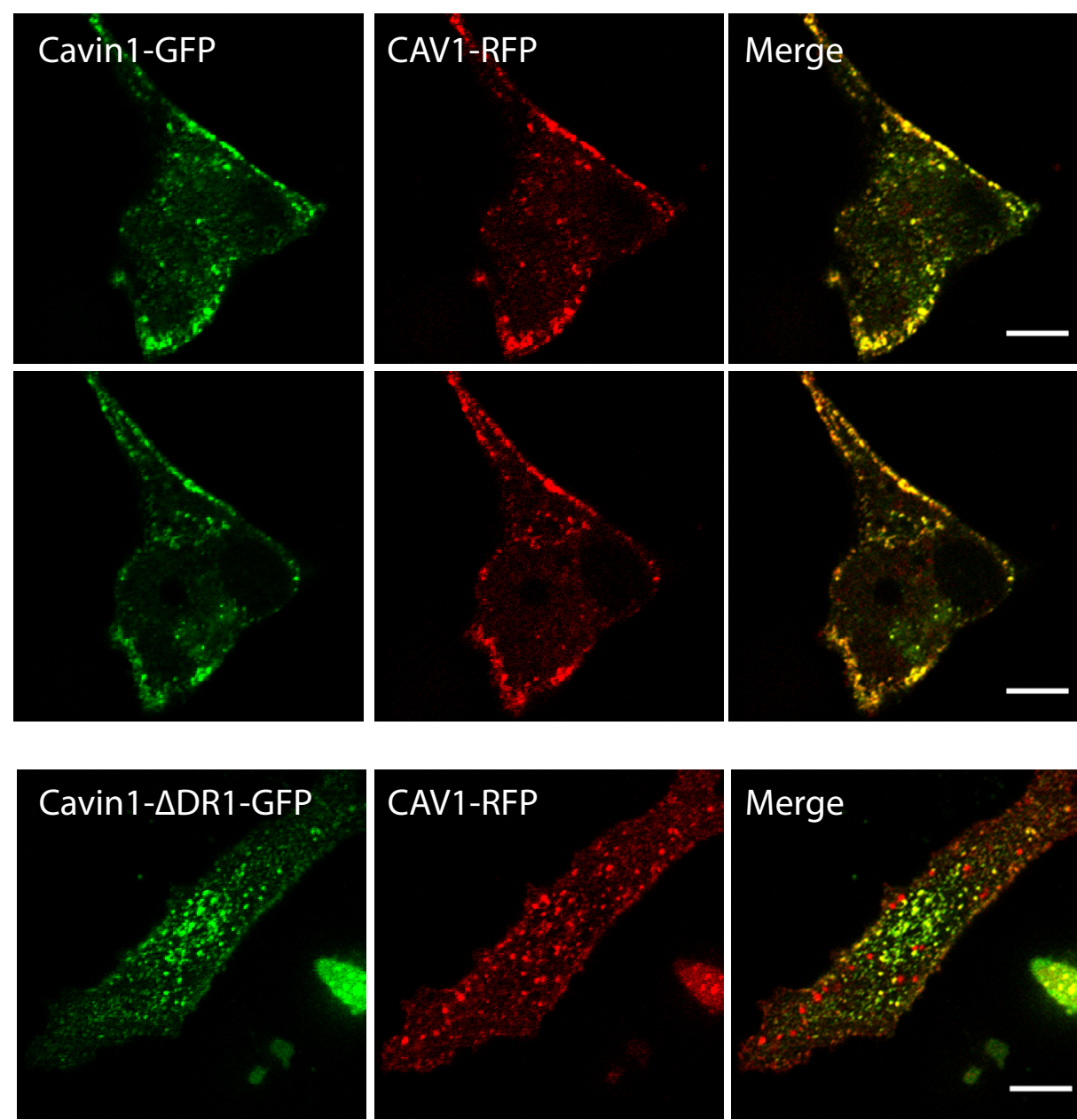

CAV1-RFP

Merge
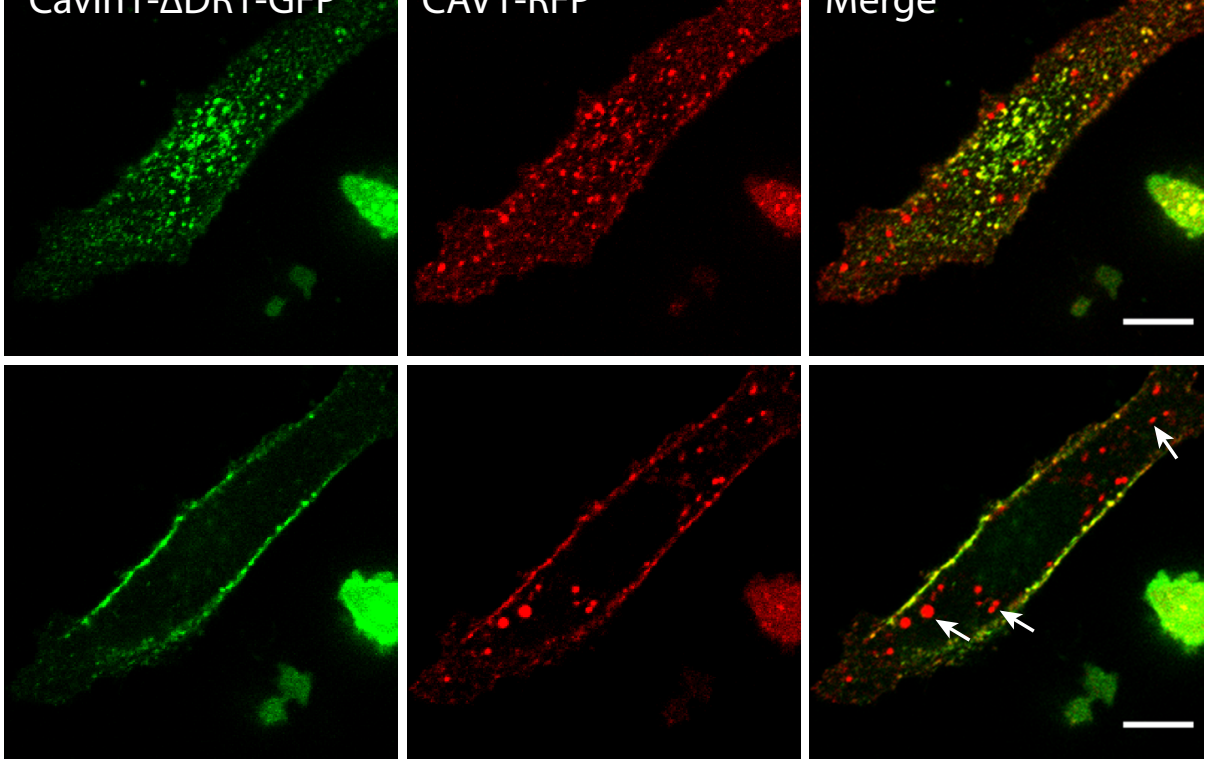